\title{
Live attenuated pertussis vaccine BPZE1 induces a broad antibody response in humans
}

\author{
Ang Lin, ${ }^{1,2}$ Danijela Apostolovic, ${ }^{1}$ Maja Jahnmatz, ${ }^{3}$ Frank Liang, ${ }^{1,2}$ Sebastian Ols, ${ }^{1,2}$ Teghesti Tecleab, ${ }^{3}$ Chenyan Wu, ${ }^{1}$ \\ Marianne van Hage, ${ }^{1}$ Ken Solovay, ${ }^{4}$ Keith Rubin, ${ }^{4}$ Camille Locht, ${ }^{5}$ Rigmor Thorstensson, ${ }^{3}$ Marcel Thalen, ${ }^{4}$ and Karin Loré ${ }^{1,2}$ \\ 'Division of Immunology and Allergy, Department of Medicine Solna, and ${ }^{2}$ Center for Molecular Medicine, Karolinska Institutet and Karolinska University Hospital, Stockholm, Sweden. ${ }^{3}$ The Public \\ Health Agency of Sweden, Stockholm, Sweden. ${ }^{4}$ LLIAD Biotechnologies, New York, New York, USA. ${ }^{5}$ University of Lille, CNRS, Inserm, CHU Lille, Institut Pasteur de Lille, Center for Infection and \\ Immunity of Lille, Lille, France.
}

\begin{abstract}
BACKCROUND. The live attenuated BPZE1 vaccine candidate induces protection against $B$. pertussis and prevents nasal colonization in animal models. Here we report on the responses in humans receiving a single intranasal administration of BPZE1. METHODS. We performed multiple assays to dissect the immune responses induced in humans $(n=12)$ receiving BPZE1, with particular emphasis on the magnitude and characteristics of the antibody responses. Such responses were benchmarked to adolescents $(n=12)$ receiving the complete vaccination program of the currently used acellular pertussis vaccine (aPV). Using immunoproteomics analysis, potentially novel immunogenic $B$. pertussis antigens were identified.
\end{abstract}

\begin{abstract}
RESULTS. All BPZE1 vaccinees showed robust $B$. pertussis-specific antibody responses with regard to significant increase in 1 or more of the following parameters: IgC, IgA, and memory B cells to B. pertussis antigens. BPZE1-specific T cells showed a Th1 phenotype, and the IgG exclusively consisted of IgG1 and IgG3. In contrast, all aPV vaccines showed a Th2-biased response. Immunoproteomics profiling revealed that BPZE1 elicited broader and different antibody specificities to $B$. pertussis antigens as compared with the aPV that primarily induced antibodies to the vaccine antigens. Moreover, BPZE1 was superior at inducing opsonizing antibodies that stimulated ROS production in neutrophils and enhanced bactericidal function, which was in line with the finding that antibodies against adenylate cyclase toxin were only elicited by BPZE1.
\end{abstract}

CONCLUSION. The breadth of the antibodies, the Th1-type cellular response, and killing mechanisms elicited by BPZE1 may hold prospects of improving vaccine efficacy and protection against $B$. pertussis transmission.

TRIAL REGISTRATION. ClinicalTrials.gov NCT02453048, NCT00870350.

FUNDING. ILIAD Biotechnologies, Swedish Research Council (Vetenskapsrådet), Swedish Heart-Lung Foundation.

\section{Introduction}

Despite high vaccination coverage worldwide, pertussis remains a major infectious disease globally in infants and young children $(1,2)$. Importantly, it has reemerged over the past decades, even in industrialized countries with sustained high vaccination rates. The reemergence of pertussis disease accelerated after the replacement of whole-cell pertussis vaccines (wPV) by acellular pertussis vaccines (aPV) in the 1990s (3-5). Although aPV confers protection against mild and severe disease, the short duration of aPV-induced immunity and inability to prevent nasal colonization and thereby reduce transmission are proposed major causes of the reemergence of pertussis

Authorship note: DA and MJ contributed equally to this work. Conflict of interest: KR is founder and CEO of ILIAD Biotechnologies. KS and MT are employees of ILIAD Biotechnologies. CL holds patents on BPZE1 vaccine and is on the Scientific Advisory Board of ILiAD Biotechnologies.

Copyright: (C) 2020, American Society for Clinical Investigation.

Submitted: November 14, 2019; Accepted: January 14, 2020; Published: March 23, 2020

Reference information: / Clin Invest. 2020;130(5):2332-2346.

https://doi.org/10.1172/JCl135020.
$(6,7)$. In addition, recent emergence of $B$. pertussis strains negative for filamentous hemagglutinin adhesion (FHA), pertussis toxin (PT), or pertactin (PRN) has been reported in countries experiencing epidemics of pertussis as a result of vaccine-induced selection (8-10). Evolution of B. pertussis to escape from aPV-induced immunity may therefore be another cause of the resurgence of pertussis (11).

Effective protection against pertussis likely requires specific antibodies and $\mathrm{T}$ cell responses in synergy with innate effector cells (12). Early clinical trials suggested the level of IgG to PT, PRN, or fimbriae 2/3 (FIM2/3) as serological correlates of protection in humans, especially after aPV vaccination $(13,14)$. However, there is still no consensus on definitive and quantitative immune correlates of protection (15-17). The polarization toward a Th1, Th2, or Th17 cellular response appears to significantly influence the quality of protective immunity (7). In animal models and humans, immunization with wPV or natural infection induces a dominant Th1 or/and Th17 response; aPV preferentially induces a Th2 response $(12,18)$. Th1 and Th17 cells proved to be more effective at conferring protection compared with Th2 cells (7), which may partly explain the lower quality of aPV-induced immunity. 
An intranasal vaccination with a live vaccine would be expected to confer effective mucosal, long-lasting protection, similar to that induced by natural infection (19). In mice, a single intranasal administration of the live attenuated vaccine candidate BPZE1 induced long-term protection against $B$. pertussis, mediated by antibodies and Th1 CD4 ${ }^{+} \mathrm{T}$ cells $(20,21)$. In baboons, BPZE1 generated full protection against disease and, importantly, against nasopharyngeal colonization upon challenge (22); aPV immunization resulted in clearance of the challenge dose from the nasopharynx at the same rate or slower than in unvaccinated naive baboons (23). The ability of BPZE1 to block colonization offers the unique prospect of blocking circulation of $B$. pertussis, thereby likely reducing pertussis disease incidence, according to epidemiological modeling (6).

BPZE1 was recently tested in Sweden in humans (ClinicalTrials.gov, NCT01188512; NCT02453048) and demonstrated good safety in healthy young adults and strong immunogenicity (24) especially using the higher doses tested (unpublished observations). In the current study, we provide more in-depth characterization of the immune responses induced by BPZE1 in human vaccinees, with particular emphasis on the Th polarization, antigen specificity, and functionality of the antibody responses. The data showed distinct differences in serum antibody breadth, specificity, and proportions of subtypes and subclasses between BPZE1and aPV-induced responses. BPZE1 induced a Th1 polarized response compared with the Th2 response induced by aPV. The BPZE1-induced antibodies further showed superior functionality compared with aPV-induced antibodies with regard to opsonization of B. pertussis, induction of ROS production, and bactericidal activity of neutrophils.

\section{Results}

Innate immune responses after BPZE1 vaccination. This study was performed in healthy adults receiving intranasal administration of $10^{9} \mathrm{CFU}$ of BPZE1. Using $10^{9} \mathrm{CFU}$, colonization of BPZE1 was detected in 10 out of the 12 vaccinees after administration (unpublished observations). Since the early innate immune activation shapes adaptive immunity, we measured the early responses after administration of BPZE1. Leukocytosis is one of the hallmarks of pertussis observed in infants, but rarely seen in adolescents or adults (25). Because of the mutation in PT, no leukocytosis is observed in naive baboons (22), so as expected, inoculation of this high dose of BPZE1 in healthy adults did not alter the frequencies of leukocytes in the blood (Supplemental Figure 1A; supplemental material available online with this article; https:// doi.org/10.1172/JCI135020DS1). In addition, no severe adverse events were reported in the BPZE1 vaccinees (unpublished observations). After vaccination, no changes were seen in the proportions of distinct monocyte subsets, CD11 ${ }^{+}$myeloid dendritic cells (MDCs), or $\mathrm{CD}_{123^{+}}$plasmacytoid dendritic cells (PDCs), nor did the expression level of activation or maturation markers on these cells change (Supplemental Figure 1, B and C). Since BPZE1 is intranasally administered, direct effects of BPZE1 on cells in the nasopharynx may not be reflected in the blood. We therefore exposed purified monocytes and neutrophils to BPZE1 in vitro and found that BPZE1 induced strong activation of both cell types evidenced by significant upregulation of CD80, CD83, CD86, CD40, CD11b, and CCR7 (Supplemental Figure 1, D and E). In addition,
BPZE1-exposed monocytes, but not neutrophils, produced the proinflammatory cytokines IL-1 $\beta$, IL-6, and TNF (Supplemental Figure 1, D and E). This suggests that BPZE1 induces rapid innate immune responses locally, but this does not lead to systemic activation of the same cell types.

Early increase of plasmablasts and activated circulating $T$ follicular helper cells. A transient increase in plasmablasts in the blood after infection or vaccination is the result of the germinal center reaction occurring during the development of antibody responses (26). The magnitude of the transient increase in plasmablasts has been shown to correlate with the magnitude and quality of the humoral response $(27,28)$. We found that the level of plasmablasts rapidly increased after BPZE1 immunization and peaked at day 14 . As expected, no plasmablast response was observed in the placebo group (Figure 1, A and B). Circulating T follicular helper (cTfh) cells, proposed as the circulating counterparts of lymphoid Tfh cells (29, 30), also increased after BPZE1 vaccination. Within the population of $\mathrm{CD} 4^{+} \mathrm{CXCR} 5^{+}$cTfh cells, there was a clear expansion of activated $\mathrm{PD}-1^{+} \mathrm{ICOS}^{+}$cells exclusively amongst the Th1-type CXCR3 ${ }^{+}$cTfh $\left(\mathrm{cTfh}_{1}\right)$ cells at 4-14 days (Figure $1, \mathrm{C}$ and D). It has been shown that the frequency of such activated $\mathrm{cTfh}_{1}$ cells correlates with the magnitude and quality of antibody responses in HCV-infected individuals or in influenza-vaccinated individuals (31-34). BPZE1-induced PD- ${ }^{+} \mathrm{ICOS}^{+} \mathrm{cTfh}_{1}$ cells also showed increased expression of activation markers compared with 2 other quiescent $\mathrm{cTfh}_{1}$ cell subsets (PD-1-1COS ${ }^{-}$and $\mathrm{PD}^{-1}{ }^{+} \mathrm{ICOS}^{-}$cells), including CD38, PD-1, CD28, and CD27 (Figure 1E). Such activated $\mathrm{cTfh}_{1}$ subsets have been linked to $\mathrm{B}$ cell helper function (33). As expected, the placebo group did not show an increase in activated $\mathrm{cTfh}_{1}$. The expansion of plasmablasts and activated $\mathrm{cTfh}_{1}$ cells therefore demonstrates that an antibody response is generated rapidly after BPZE1 immunization.

Induction of B. pertussis-specific antibodies by BPZE1 vaccination. Since BPZE1 expresses the full spectrum of B. pertussis antigens, we measured the levels of antibodies against the entire bacteria, using the BPZE1 lysate as coating antigens in the ELISA. At 28 days after immunization, BPZE1 vaccinees had significantly increased total IgG and IgA titers against BPZE1 lysates (Figure 2A). Amongst the 12 immunized individuals, 10 subjects showed a 1.5 -fold or greater induction of BPZE1-specific IgG or IgA, with 6 of them showing a 1.5-fold or greater induction of both antibody isotypes (Supplemental Figure 2A). Interestingly, the 2 subjects showing no detectable nasopharyngeal colonization after vaccination also mounted robust IgG and IgA titers. BPZE1-specific total IgG titers remained higher than prevaccination levels at 6 and 12 months, indicating a durable vaccine response, as also reported in mouse studies (21, 35). Although IgA waned faster than IgG, both were still elevated at 6 months after vaccination. The total IgG and IgA responses to BPZE1 and the recent clinical isolate BP611-98 were almost identical, showing that the BPZE1-induced antibodies are highly cross-reactive (Figure 2, B and C; and Supplemental Figure 2C). This observation is also supported by the high similarity in protein antigen composition between BPZE1 and BP611-98, as determined by 2D PAGE (Figure 2D). In the placebo group, the levels of IgG and IgA did not change over time (Supplemental Figure 2, B and D).

BPZE1 induces a Th1-biased $C D 4^{+} T$ cell response. As mentioned above, in addition to the antibody response, the quality of the Thelper cell response is critical in the induction of protection against per- 
A

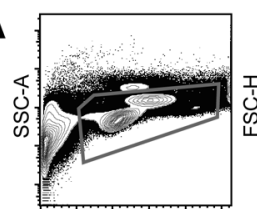

FSC-A

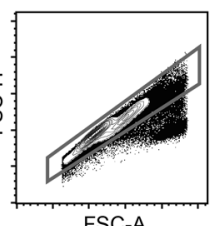

FSC-A

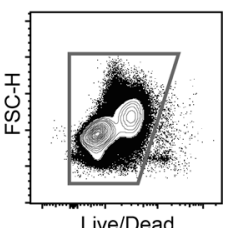

Live/Dead

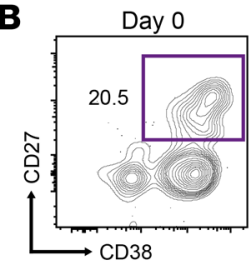

C
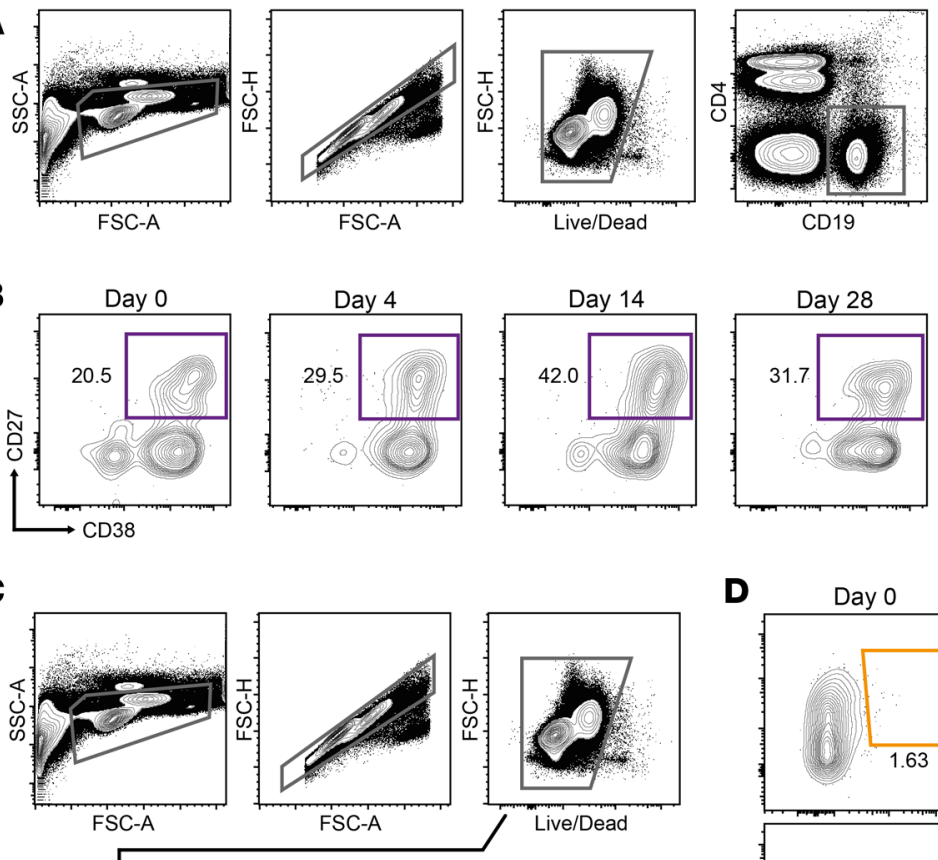

CD19
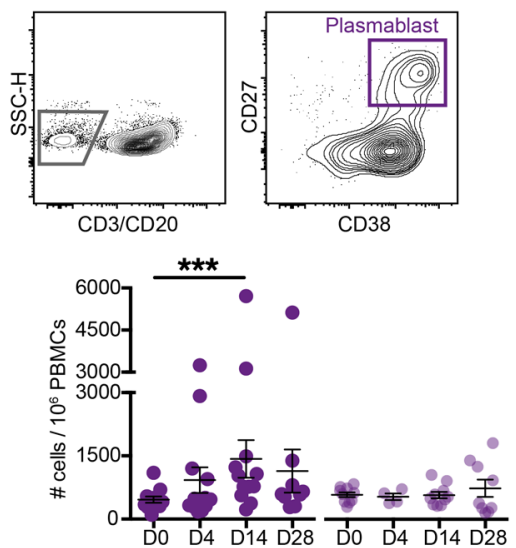

D
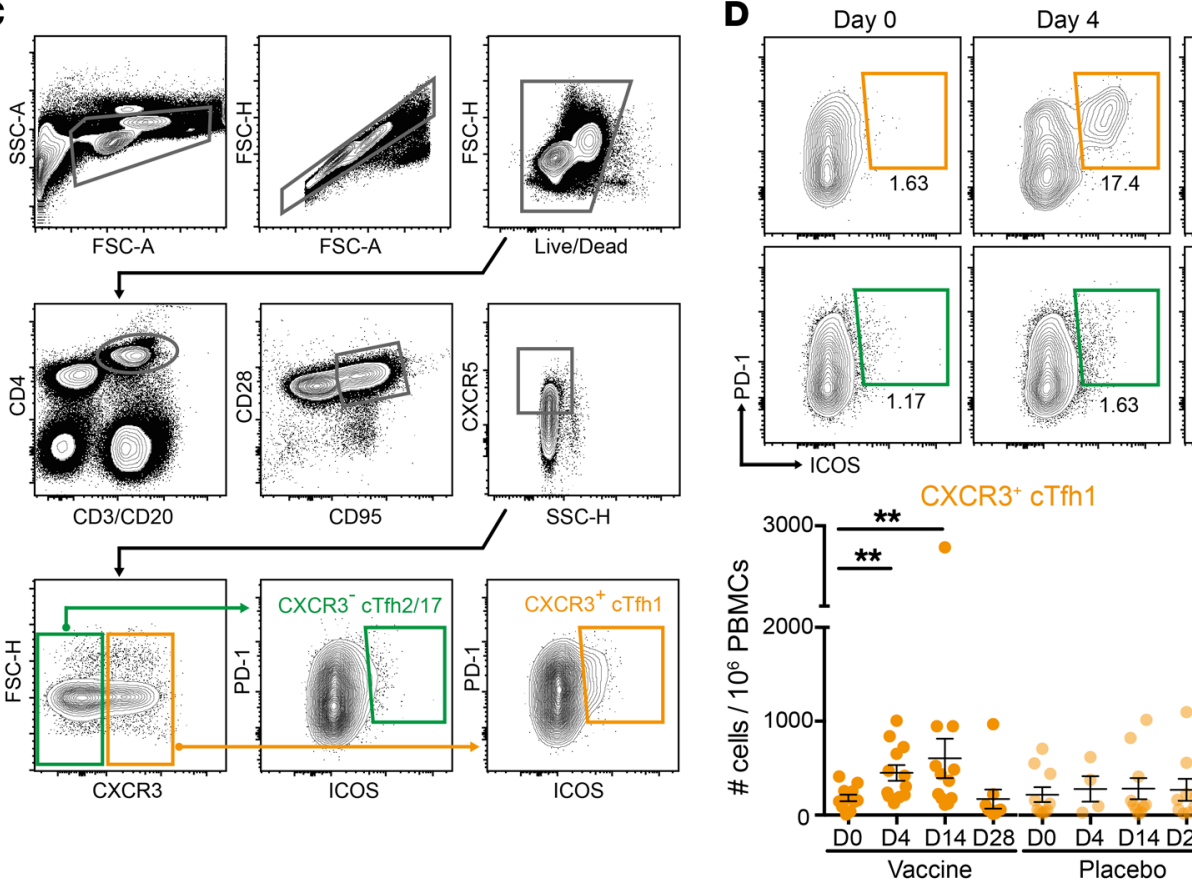

- Vaccine

- Placebo

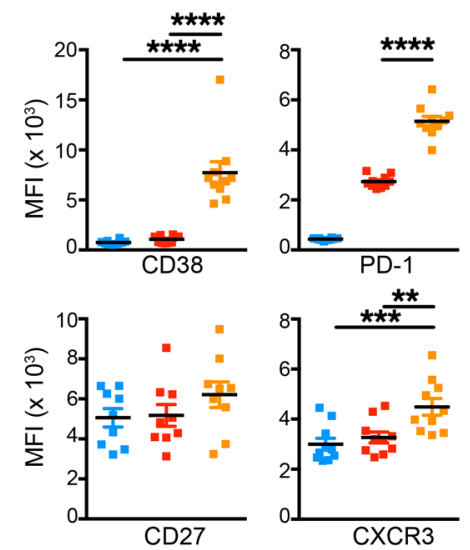

E
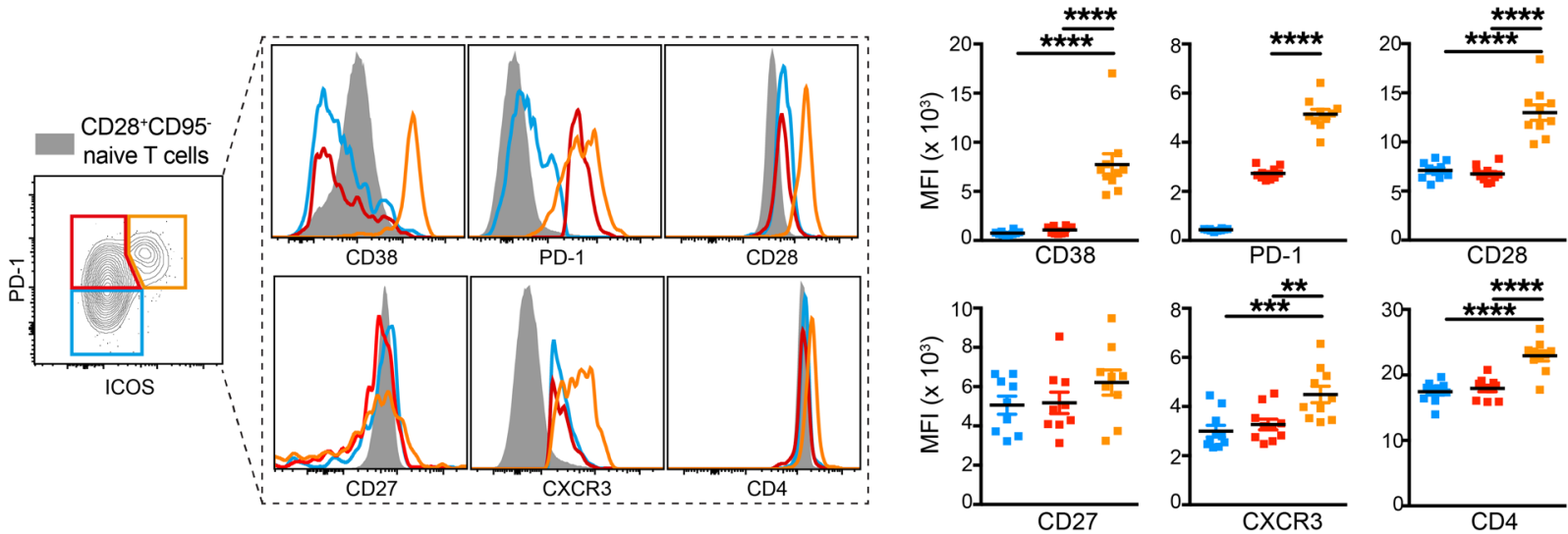

Figure 1. Expansion of plasmablasts and activated cTfh1 cells following BPZE1 vaccination. (A) Phenotypic identification of circulating plasmablasts according to the depicted gating strategy. (B) Frequency of plasmablasts at different time points following BPZE1 vaccination. Data from one representative BPZE1 vaccinee are shown. Dot plots show number of plasmablasts per million PBMCs. (C) Phenotypic identification of activated cTfh cells according to the depicted gating strategy. (D) Frequency of activated cTfh1 and cTfh2/17 cells at different time points following BPZE1 vaccination. Data from one representative BPZE1 vaccinee are shown (upper panel). Dot plots show number of cells per million PBMCs (lower panel) $(n=12$ for BPZE1 group and $n=10$ for placebo group). (E) Surface expression of indicated markers on PD1+ICOS+, PD1+ICOS-, and PD1-ICOS- cTfh1 cells. Mean fluorescence intensity (MFI) values of markers are shown. Two-tailed Wilcoxon matched-pairs signed-rank test was used for all compiled data. ${ }^{* *} P \leq$ $0.01,{ }^{* *} P \leq 0.001,{ }^{* * *} P \leq 0.0001$. 
A
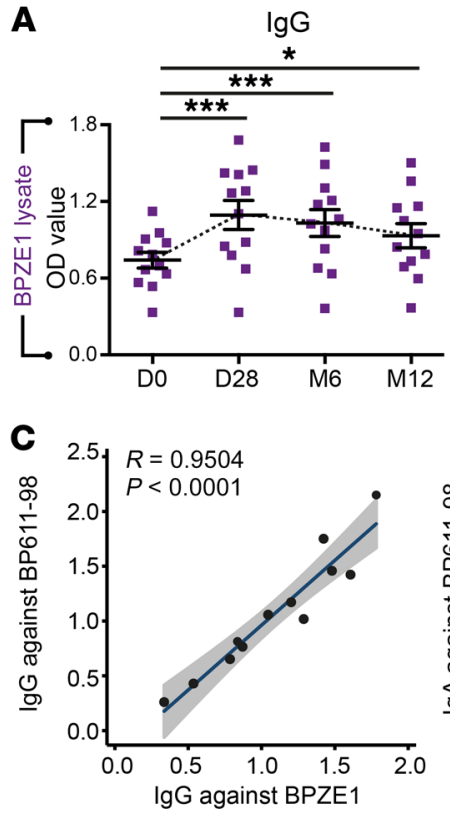

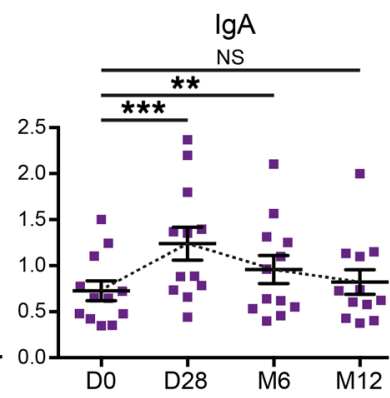

B
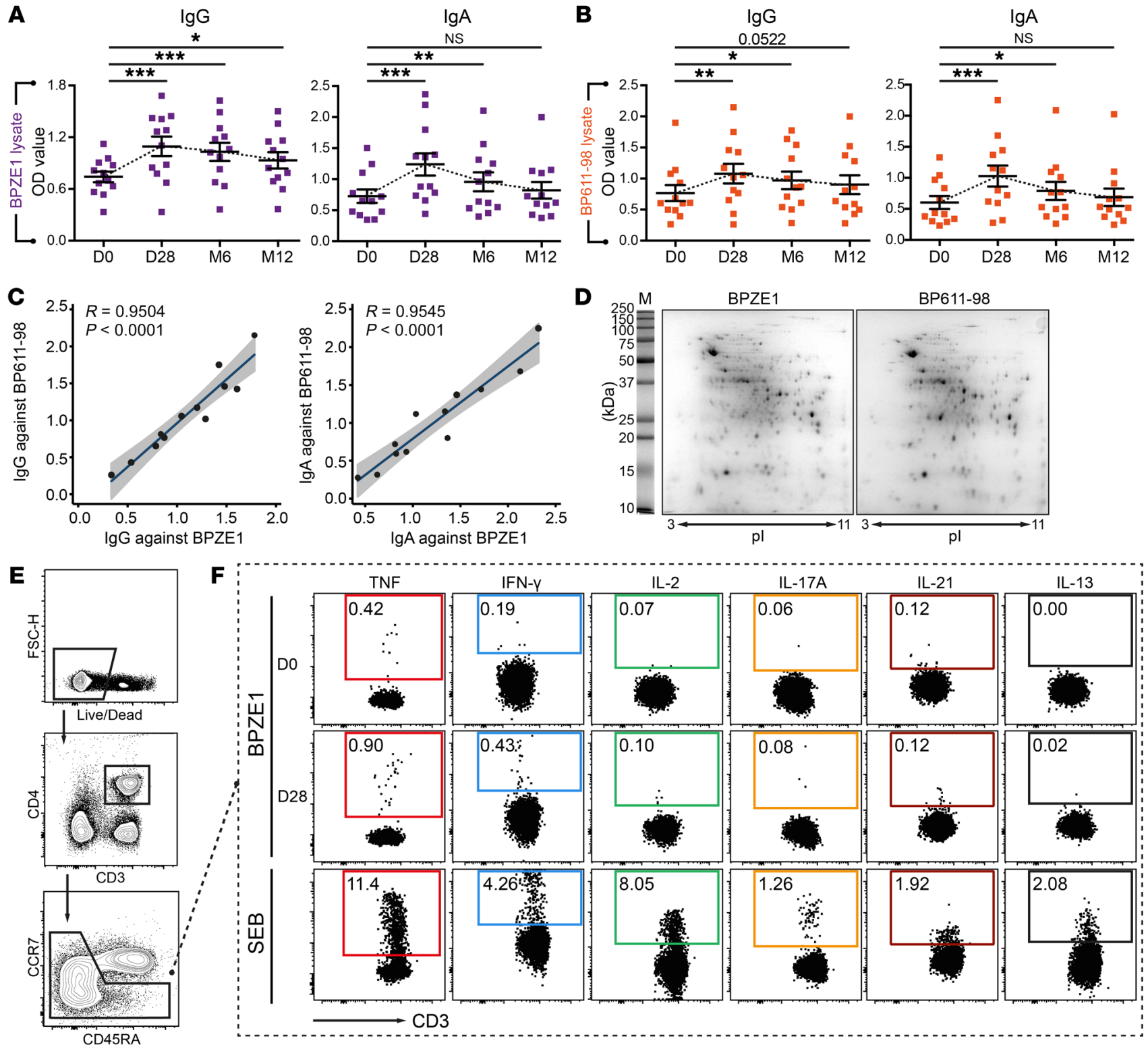

G

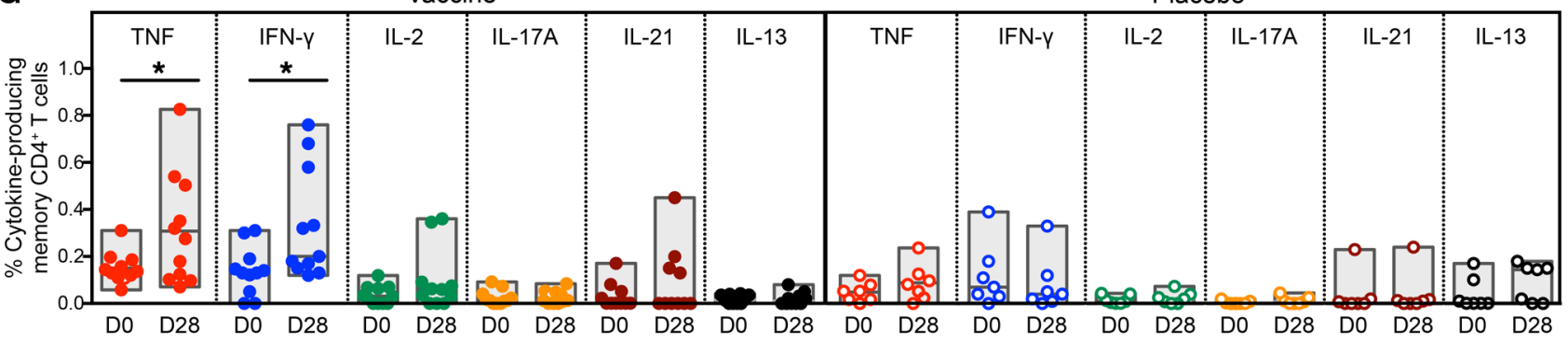

Figure 2. BPZE1 vaccination induced robust antibody titers and Th1-biased CD4+ $\mathbf{T}$ cell responses. (A and B) Antibody responses directed against BPZE1 or BP611-98 lysates in BPZE1 vaccinees $(n=12)$ at different time points after vaccination. Wilcoxon matched-pairs signed-rank test was used. (C) Correlation of IgG or IgA responses directed against BPZE1 lysates with that directed against B611-98 lysates. Pearson's correlation analysis was used. (D) Protein fingerprints of BPZE1 and BP611-98 by 2D PAGE. Gels were stained with colloidal Coomassie Brilliant Blue (CBB) G-250. Four independent experiments were performed and one representative gel is shown. (E) Representative gating of CD4+ memory T cell populations. (F) PBMCs collected before and at 28 days after vaccination from BPZE1 vaccinees $(n=11)$ or placebo subjects $(n=7)$ were stimulated with heat-inactivated BPZE1 (bacteria/cell ratio $=10: 1)$. Cells treated with Staphylococcus enterotoxin B were used as a positive control. Levels of the indicated cytokine-producing memory CD4 ${ }^{+} T$ cells were analyzed Data from one representative donor are shown. (C) Quantification of cytokine-producing CD4+ memory T cells following heat-inactivated BPZE1 stimulation in BPZE1 vaccinees $(n=11)$ and placebo subjects $(n=7)$. Negative control (PBS) values were subtracted. Two-tailed paired $t$ test was used. ${ }^{*} P \leq 0.05$, ${ }^{*} P \leq 0.01,{ }^{* *} P \leq 0.001$. 

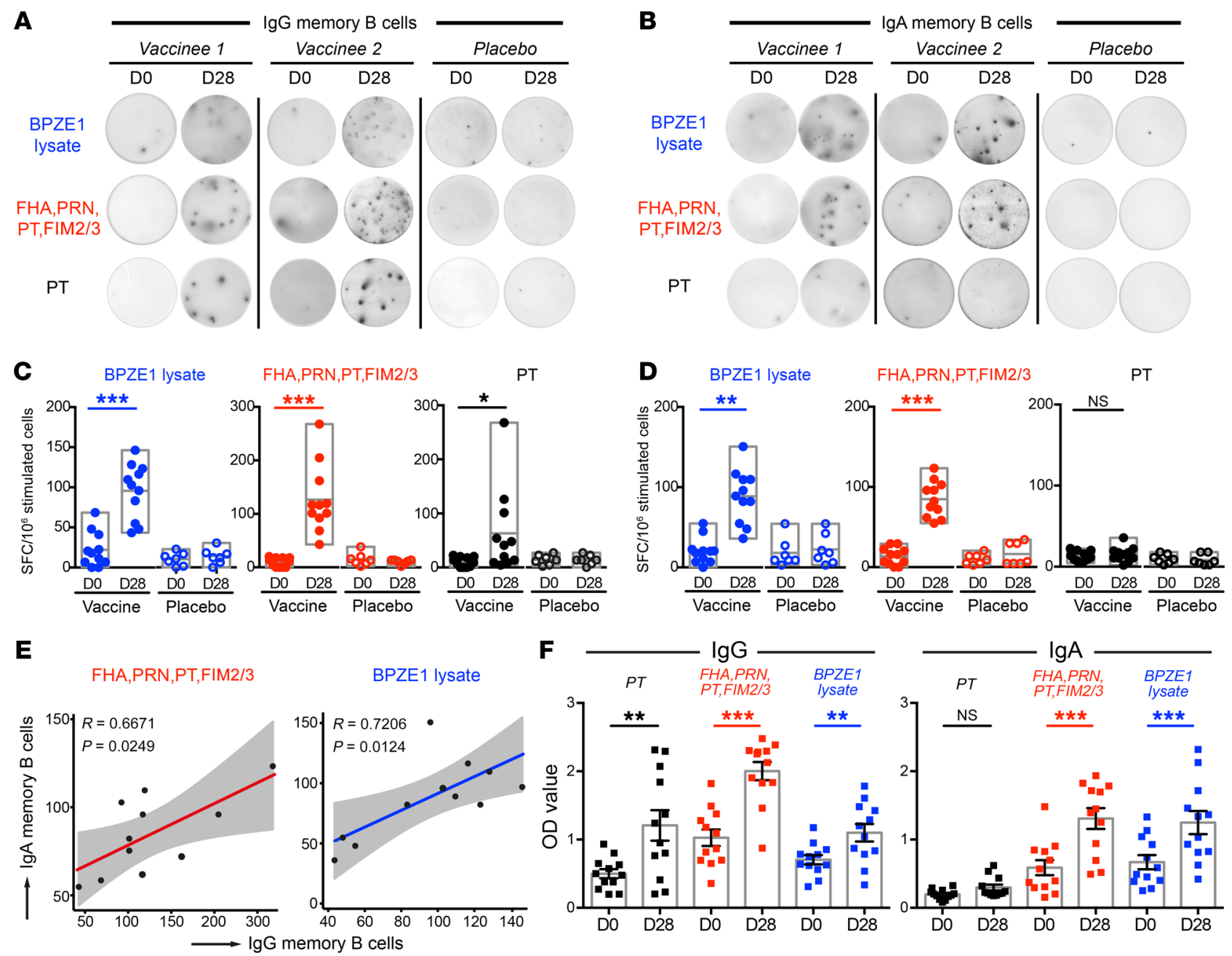

Figure 3. BPZE1 vaccination induced strong memory B cell responses. (A and B) IgG or IgA memory B cell responses targeting BPZE1 lysates, antigen mix (FHA, PT, PRN and FIM2/3), or PT were evaluated by ELISpot. Data from 2 representative BPZE1 vaccinees and 1 placebo subject are shown. (C and D) Magnitudes of specific IgG or IgA memory B cell responses determined by ELISpot are enumerated as spot-forming cells (SFC) per million stimulated PBMCs. Vaccine group $(n=11)$, placebo group $(n=7)$. Two-tailed Wilcoxon matched-pairs signed-rank test was used. (E) Correlation of IgG and IgA memory B cell responses directed against antigen mix or BPZE1 lysates. Pearson's correlation analysis was used. (F) IgG and IgA titers targeting PT, antigen mix, or BPZE1 lysates were evaluated in BPZE1 vaccinees $(n=12)$. Two-tailed Wilcoxon matched-pairs signed-rank test was used. ${ }^{*} P \leq 0.05,{ }^{* *} P \leq 0.01,{ }^{* * *} P \leq 0.001$.

tussis (36). The expansion of activated cTfh ${ }_{1}$ cells and not of $\mathrm{cTfh}_{2 / 17}$ cells in the BPZE1 vaccinees suggests that BPZE1 primarily induces a Th1-polarized $\mathrm{T}$ cell response. Consistently, a dominant Th1-biased $\mathrm{CD}^{+} \mathrm{T}$ cell response was found, as evidenced by a majority of the BPZE1-specific T cells producing TNF- or IFN- $\gamma$. No or very limited numbers of Th2 (IL-13) or Th17 (IL-17A) cells were detected (Figure 2, E-G). A proportion of BPZE1-specific CD $4^{+} \mathrm{T}$ cells expressed more than 1 cytokine indicating polyfunctionality (Supplemental Figure 3). The placebo group showed no induction of BPZE1-specific T cells (Figure 2G). Although animal studies have shown that BPZE1 can also induce Th17 cells apart from Th1 cells, the Th17 responses were restricted to the lung, spleen, or nasal cavity $(35,37)$. In addition, in wPV-immunized mice, vaccine-specific Th17 responses were only detectable in the lung or nasal cavity, not in the blood (38). Therefore, whether BPZE1 in humans is able to elicit a Th17 response in mucosal tissues merits further investigation.

Although early studies indicated that $\mathrm{CD}^{+} \mathrm{T}$ cells are not necessary for protection against pertussis $(39,40)$, recent data proposed a role for cytotoxic $\mathrm{CD}^{+} \mathrm{T}$ cells, especially due to the intracellular infection properties of $B$. pertussis $(41,42)$. However, in contrast to the $\mathrm{CD} 4^{+} \mathrm{T}$ cells, no induction of detectable BPZE1specific $\mathrm{CD}^{+} \mathrm{T}$ cells were found in the vaccinees (data not shown).

Induction of robust vaccine-specific memory $B$ cell responses by $B P Z E 1$. We next evaluated the frequencies of memory B cell populations specific to BPZE1 lysate, a mix of PT, FHA, PRN, and FIM2/3, as well as PT alone. All vaccines showed a significant IgG memory B cell response to the BPZE1 lysate, as well as to the antigen mix, and the majority of individuals also showed PT-specific memory B cell responses (Figure 3, A and C). IgA memory B cells were also detected in all vaccinees and recognized BPZE1 lysate and antigen mix, but not PT (Figure 3, B and D). The levels of IgG memory B cells correlated with the frequencies of IgA memory B cells for the antigen mix and the lysate (Figure 3E). In agreement with the memory B cell data, the serum IgG recognized PT, the antigen mix, and the whole lysates; the serum IgA did not react with PT, but did react with the antigen mix and BPZE1 lysates (Figure 3F). The lack of PT-specific 
A Immunization with BPZE1:

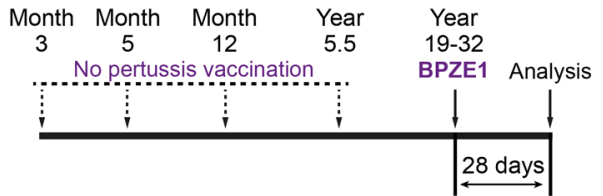

Immunization with aPV:

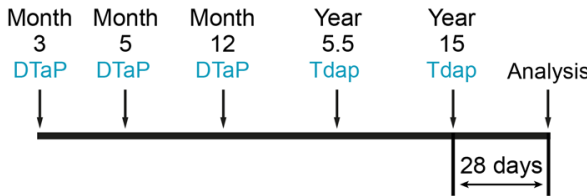

B

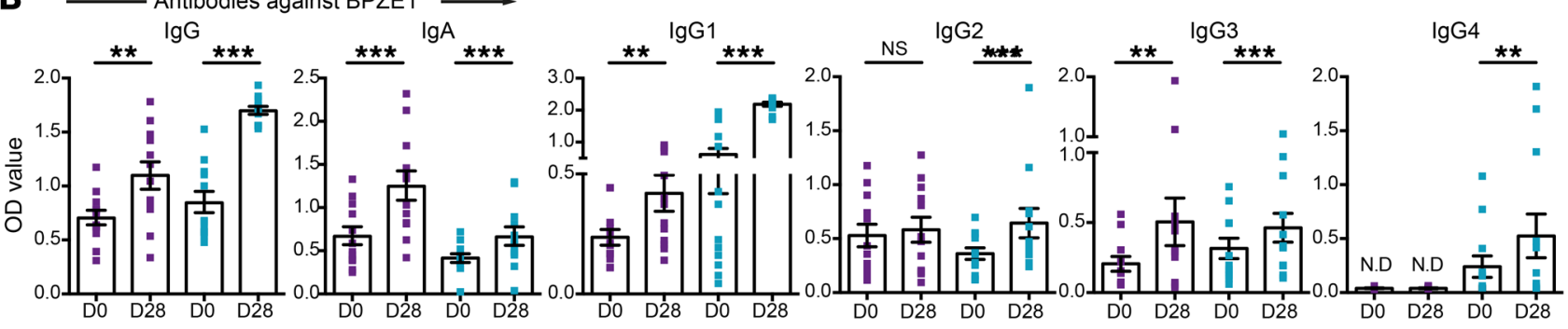

C
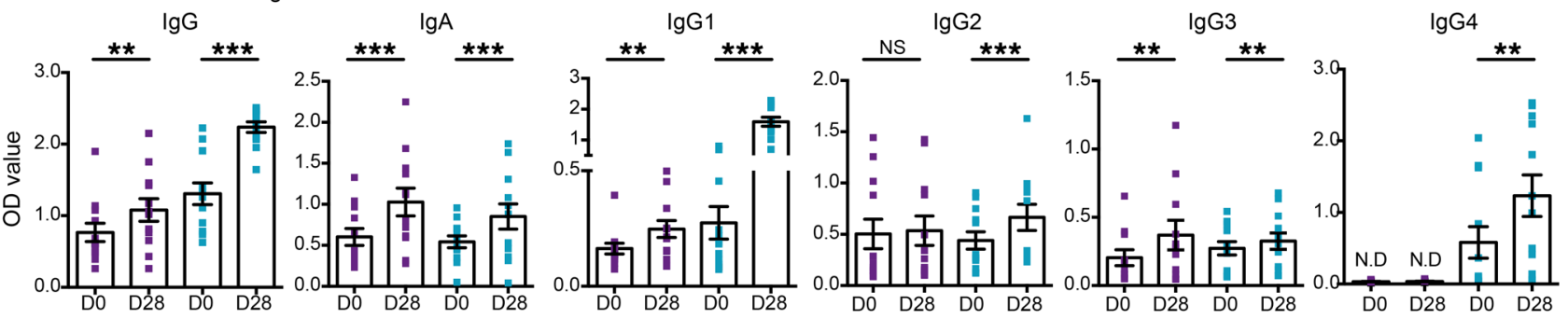

D - Antibodies against FHA,PT,PRN,FIM $\longrightarrow$ $\lg G$
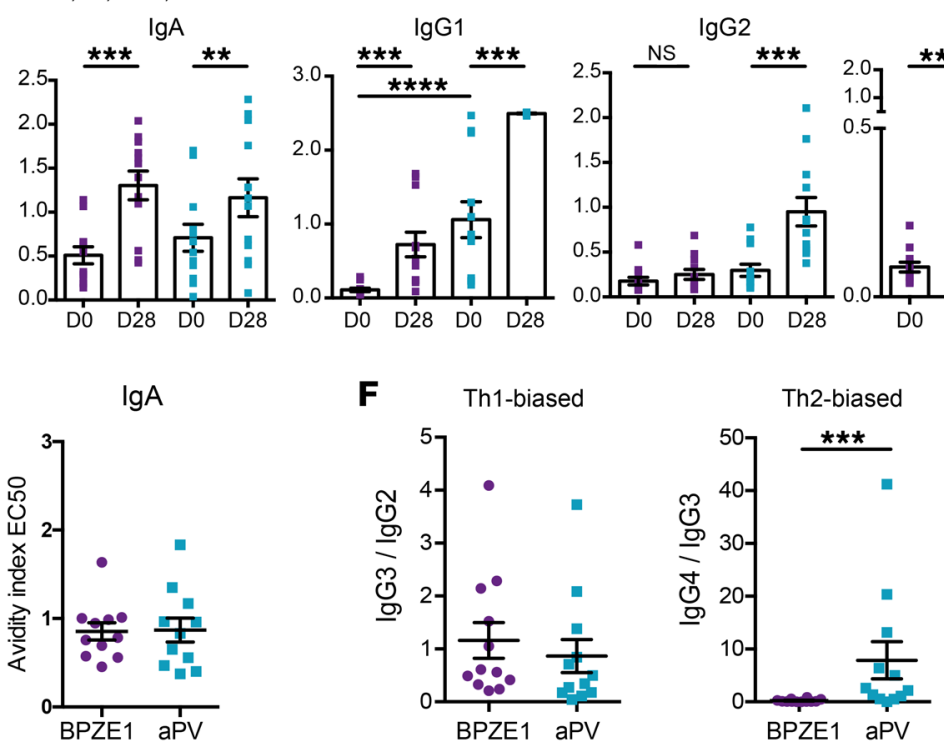

$\operatorname{lgG3}$

$\operatorname{lgG} 4$

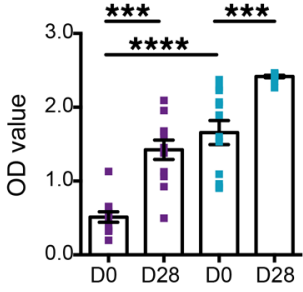

$\lg \mathrm{A}$
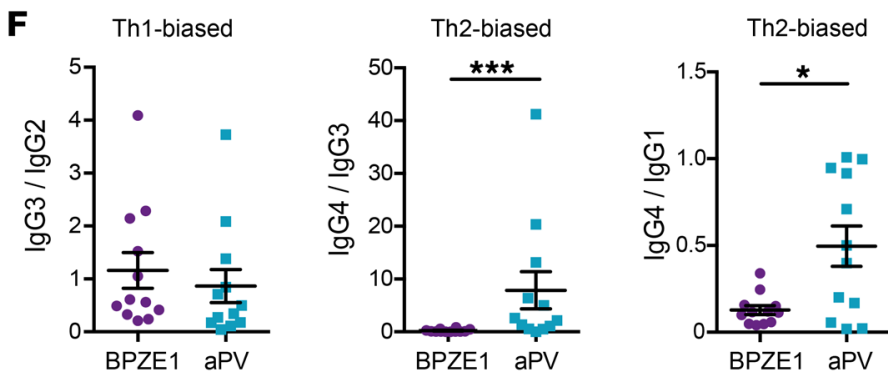

Figure 4. Different types of antibody responses were elicited by BPZE1 and aPV immunization. (A) Immunization schedule for BPZE1 vaccinees ( $n=12)$ and aPV vaccinees $(n=12)$. (B-D) Antibody titers of IgG, IgA, and 4 IgG subclasses directed against BPZE1 lysates, BP611-98 lysates, and antigen mix in BPZE1 and aPV vaccines were evaluated. OD value is shown. Two-tailed Wilcoxon matched-pairs signed-rank test was used for comparison in the same group. Two-tailed Mann-Whitney test was used for comparison between 2 groups. (E) Antibody avidity of IgG and IgA targeting BPZE1 lysates in BPZE1 and aPV vaccines was evaluated. Avidity index EC50 represents the concentration of chaotropic agent $\left(\mathrm{NH}_{4} \mathrm{SCN}\right)$ that dissociates $50 \%$ of the antibody-antigen binding. Two-tailed Mann-Whitney test was used. (F) Ratio of IgG3/IgG2, IgG4/lgG3, and IgG4/lgG1 against BPZE1 lysates at 28 days after vaccination in BPZE1 and aPV vaccinees is shown. Mann-Whitney test was used. ${ }^{*} P \leq 0.05,{ }^{* *} P \leq 0.01,{ }^{* * *} P \leq 0.001,{ }^{* * * *} P \leq 0.0001$.

IgA memory B cells may be a consequence of the slow onset of PT expression during the colonization process in the nasal cavity, unlike FHA, PRN, and FIM, which are fully expressed during initial stages of colonization (43). In any event, these data suggest that B. pertussis antigens other than PT in BPZE1 do elicit an IgA response.
Differences between the BPZE1- and aPV-induced antibody responses. In order to further characterize the Th polarization and antibodies, we benchmarked the responses in the BPZE1 vaccinees to responses induced by aPV immunization (Figure $4 \mathrm{~A})$. Antibody responses generated 28 days after the fifth vac- 
A

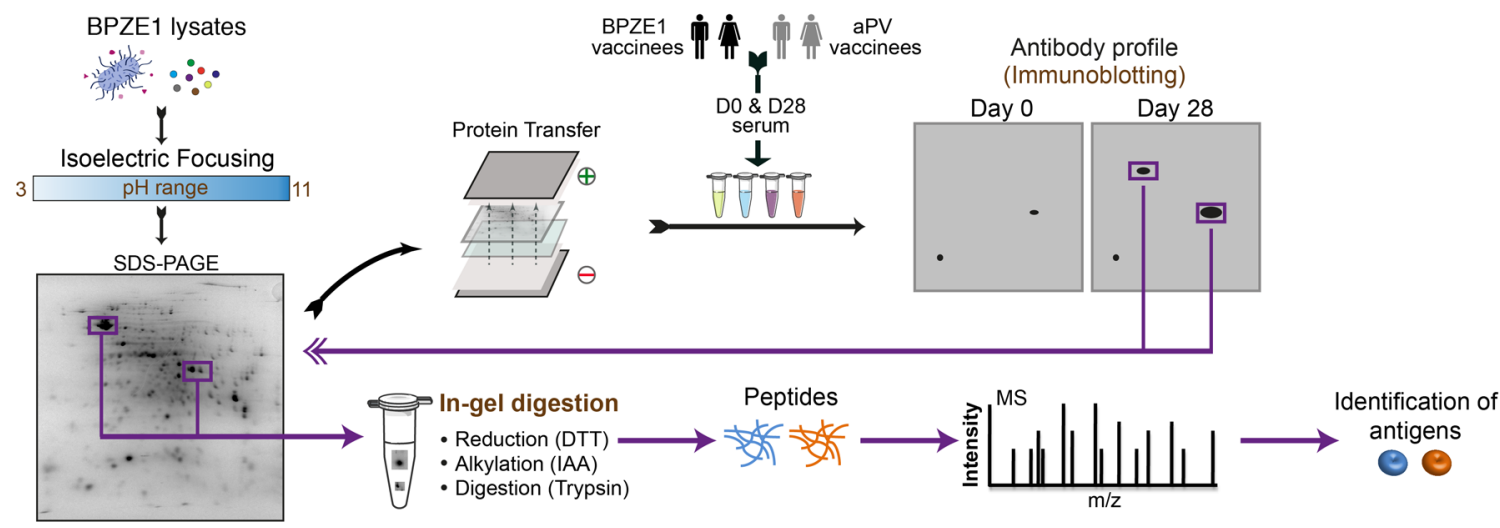

B
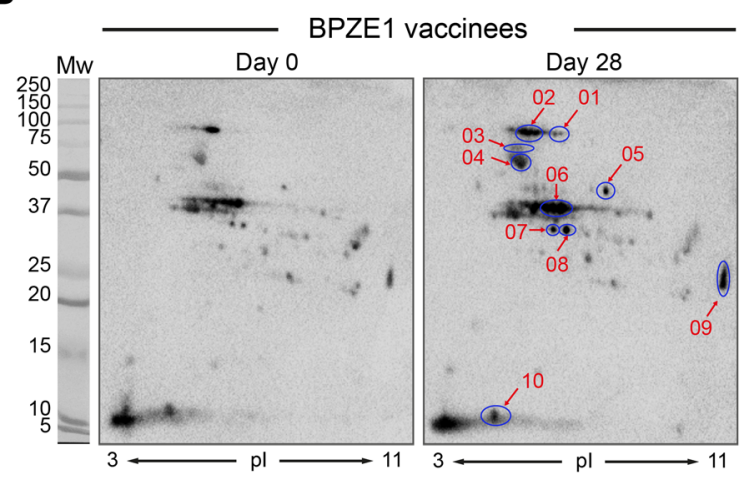

C
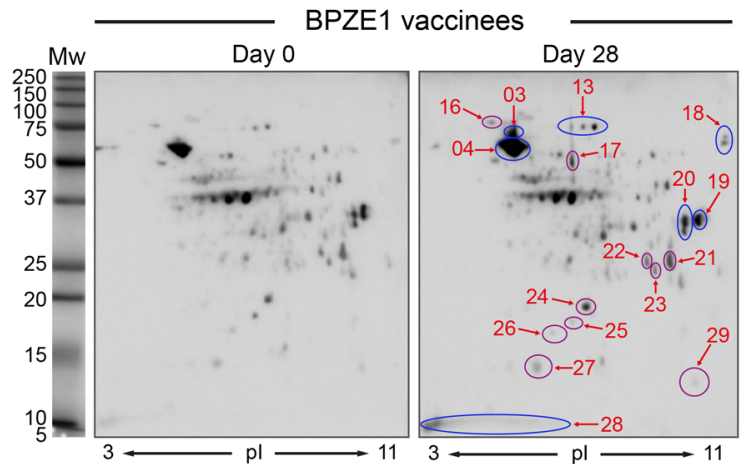

D

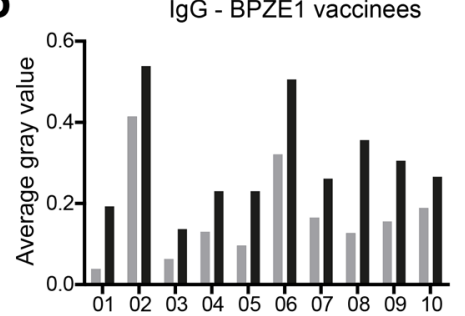

IgG - aPV vaccinees

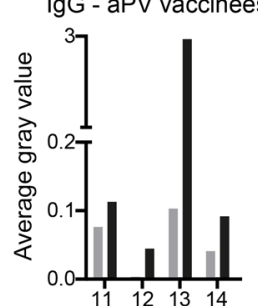

$\lg G$

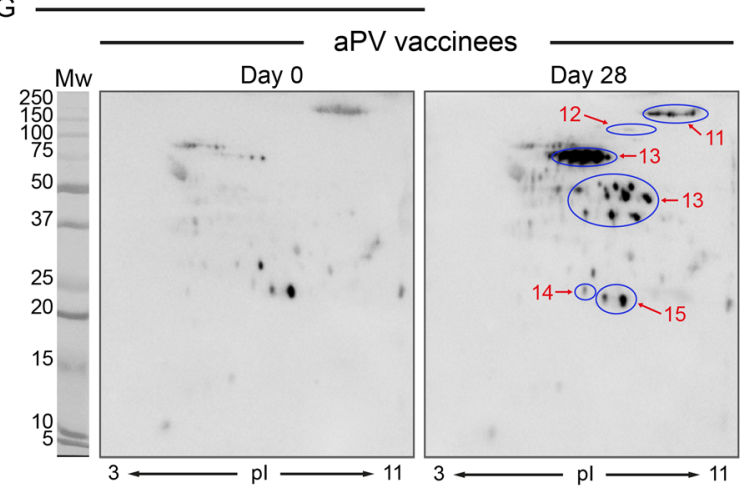

$\lg A$

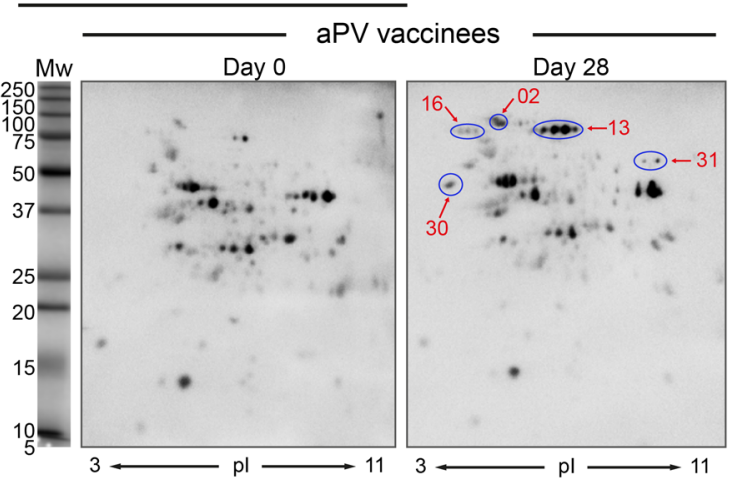

IgA - BPZE1 vaccinees

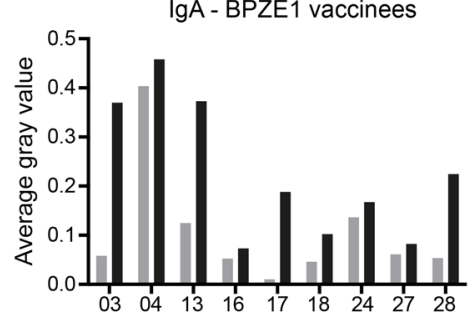

Figure 5. Immunoproteomics profiling of antibody responses in BPZE1 and aPV vaccines and identification of potentially novel immunogenic antigens. (A) Experiment design of $2 \mathrm{D}$ immunoblotting to evaluate antibody responses in BPZE1 and aPV vaccinees and identification of antigens by LC-MS/MS. (B and C) BPZE1 lysates were separated by 2D PACE followed by immunoblotting analysis with pooled serum of 12 vaccinees from BPZE1 or aPV group. Profiles of IgC and IgA responses were evaluated. Two or 3 independent experiments were performed with similar results. One representative blot is presented. (D) Intensity of the indicated spots was quantified using Delta-2D software. Average gray value is shown.

cination with aPV were compared with the responses induced 28 days after a single immunization with BPZE1. Both groups showed a significant increase in IgG and IgA titers to the BPZE1 lysate, the clinical strain BP611-98, as well as the antigen mix of FHA, PT, PRN, and FIM after immunization (Figure 4, B-D). The total IgG levels were overall higher in the aPV than in the BPZE1 vaccinees, likely due to the repeated immunizations with aPV these individuals have received, which was also reflect- 


\section{Table 1. Identification of immunogenic B. pertussis antigens within BPZE1}

\begin{tabular}{|c|c|c|}
\hline Label & UniProt ID & Protein/gene name \\
\hline 01 & Q7VZUO & PNPase \\
\hline 02 & Q45340 & BrkA autotransporter \\
\hline 03 & Q7VZGO & $30 \mathrm{~S}$ ribosomal protein $\mathrm{S} 1$ \\
\hline 04 & P48210 & GroEL \\
\hline 05 & P14283 & Pertactin autotransporter \\
\hline \multirow[t]{3}{*}{06} & Q7VU66 & $\operatorname{TrpD}$ \\
\hline & Q7VYN1 & Liv) \\
\hline & Q7VVC9 & Putative $A B C$ transport soluble-binding protein \\
\hline 07 & Q7VZBO & CbpA \\
\hline 08 & Q7VUV7 & Putative exported soluble-binding protein \\
\hline 09 & Q7W0S2 & RplA \\
\hline \multirow[t]{2}{*}{10} & Q7W0E4 & GcvH \\
\hline & Q7VW32 & ACP \\
\hline 11 & $\mathrm{P} 12255$ & FHAb \\
\hline 12 & Q7VVI2 & FHAs \\
\hline 13 & P14283 & Pertactin autotransporter \\
\hline 14 & P04977 & PT S1 \\
\hline \multirow[t]{2}{*}{15} & P04978 & PT S2 \\
\hline & P04979 & PT S3 \\
\hline 16 & Q7VVY2 & Chaperone protein DnaK \\
\hline 17 & Q7VUE7 & Wbp0 \\
\hline 18 & P12255 & FHAb \\
\hline 19 & Q7VWW8 & Putative exported protein \\
\hline 20 & Q7VXB3 & Putative exported protein \\
\hline $21 / 22$ & P40415 & PPlase \\
\hline 23 & Q7VYH5 & Putative amino acid $A B C$ transporter \\
\hline \multirow[t]{2}{*}{24} & P37369 & SODb \\
\hline & Q7W0K2 & DsbA \\
\hline 25 & Q7W0S4 & NusG \\
\hline 26 & Q7VX98 & PpiB \\
\hline 27 & P0A339 & GroES \\
\hline 28 & & LPS \\
\hline 29 & POA3R5 & PT S4 \\
\hline 30 & Q7VW79 & Enolase \\
\hline 31 & Q7VSR6 & Putative extracellular solute-binding protein \\
\hline
\end{tabular}

ed in the high baseline levels of antibodies against the antigen mix (Figure 4D). In contrast to the high IgG levels, the aPV vaccinees showed lower or similar IgA levels, both prior to and after immunization, compared with the BPZE1 vaccinees (Figure 4B). This is in line with the inability of aPV to induce IgA responses in naive mice $(37,44)$, which means that aPV-induced IgA in humans may primarily be a boost effect of previous subclinical exposure to circulating $B$. pertussis (45). In contrast, preexisting immunity both in terms of IgG and IgA in the BPZE1 vaccinees would exclusively stem from natural exposure. Measurement of the binding strength (avidity and affinity) of the vaccine-induced $\operatorname{IgG}$ and IgA after normalizing the antibody levels (OD value $=1$ ) showed that aPV-induced IgG had higher binding strength than BPZE1-induced IgG, suggesting that the repeated immunizations resulted in an increased binding quality of antibodies (Figure $4 \mathrm{E}$ ). There was no such increase for IgA, again likely because $\mathrm{aPV}$ is not efficient at inducing IgA responses.
Furthermore, the increase of total IgG in the BPZE1 vaccinees was exclusively contributed by IgG1 and IgG3, consistent with their Th1-biased $\mathrm{T}$ cell response. No increase in IgG2 was observed, whereas IgG4 was not detectable (Figure 4, B-D). In contrast, the aPV induced all 4 IgG subclasses. The IgG2 and IgG4 responses exclusively detected in the aPV group indicate a Th2skewed response compared with the BPZE1 group (Figure 4, B-D), as previously reported $(46,47)$. This is also demonstrated by the ratios of different IgG subclasses (Figure $4 \mathrm{~F}$ ). As expected, the levels of different IgG subclasses in the placebo subjects did not change (Supplemental Figure 2).

Immunoproteomics profiling of antibody responses induced by $B P Z E 1$ and $a P V$. Apart from the differently polarized responses to BPZE1 versus aPV, antigens other than FHA, PT, PRN, and FIM included in aPV may confer better protection $(21,37)$. By using a mass spectrometric analysis, we obtained a full proteome map of BPZE1 with 1599 proteins identified (Supplemental Table 2). Using gene ontogeny (GO) enrichment analysis, the identified proteins were categorized and annotated based on their molecular function (MF), cellular component (CC), and biological process (BP) (Supplemental Figure 4A). Functional categories of the proteins were determined using the KEGG Brite database. The most represented proteins were from the cytoplasm cellular compartment category and with a role in the biosynthesis of secondary metabolites, antibiotics, and amino acids (Supplemental Figure 4B). Analysis of the proteome map of BP611-98 identified 1494 proteins (data not shown), of which 1410 were shared with BPZE1 (Supplemental Figure 4C), supporting their similarity in protein composition. The analysis also confirmed the absence of FIM3 and of dermonecrotic toxin (DNT) in BPZE1 $(20,48)$ (Supplemental Figure 4C). In addition, as a circulating virulent strain, BP61198 contained FIM3 and DNT, but not FIM2 and adenylate cyclase toxin (ACT). Other immunogenic antigens such as PT, FHA, and PRN were present in both BPZE1 and BP611-98.

We combined 2D immunoblotting with LC-MS/MS analysis to provide an in-depth characterization of the antigen-binding specificities of the antibodies induced in BPZE1- versus aPV-immunized individuals (Figure 5A). Prior to vaccination, the BPZE1 group showed IgG directed to a higher number of antigens than the aPV group (Figure 5B, left panels), suggesting that although the majority of the subjects in BPZE1 group did not receive pertussis vaccination during childhood, they had preexisting immunity acquired by natural exposure to $B$. pertussis, as also detected by ELISA (Figure 2 and Figure 4). In contrast, the aPV group showed a restricted response of baseline IgG, essentially corresponding to the antigen components of aPV. After vaccination, the IgG response induced by BPZE1 also showed a much broader antigen recognition repertoire than that induced by aPV. In both groups, there was a booster effect of the specific responses detected at baseline. The antigen recognition repertoires of the IgA responses both at baseline and after vaccination were more similar between the BPZE1 and aPV groups than that observed for IgG (Figure 5C). This may again be due to priming of IgA responses by natural exposure in both groups.

Identification of potentially novel antigens by LC-MS. The intensities of the spots before and after immunization were quantified using Delta-2D software (Figure 5D), measured as the average gray value. In total, 31 protein spots that showed increased inten- 
Table 2. Reported immunogenicity of the identified B. pertussis antigens in this study

\begin{tabular}{|c|c|c|c|c|c|c|c|}
\hline UniProt ID & Gene locus ${ }^{B}$ & Protein name/function & $\begin{array}{l}\text { Subcellular } \\
\text { localization }\end{array}$ & $\begin{array}{c}\text { Experimental } \\
\text { model }\end{array}$ & Immunogenicity ${ }^{D}$ & Protective capacity & Reference \\
\hline Q45340 & ВР3494 & $\begin{array}{l}\text { BrkA, inhibits the classical } \\
\text { complement pathway }\end{array}$ & OM & Mice & + & - & $44,50,52,63$ \\
\hline Q7VZUO & BP0795 & $\begin{array}{l}\text { PNPase, Polyribonucleotide } \\
\text { nucleotidyltransferase }\end{array}$ & C & Rabbit & + & unknown & 50 \\
\hline Q7VYN1 & BP1285 & Leu/ile/val-binding protein & $P$ & Mice & + & unknown & 52 \\
\hline Q7WOS2 & ВР0011 & RplA, 50 S ribosomal protein L1 & C & Rabbit & + & unknown & 50 \\
\hline Q7VVY2 & BP2499 & $\begin{array}{l}\text { Dnak, chaperone protein; } \\
\text { Heat shock protein } 70\end{array}$ & C & Mice, Human & + & unknown & 51,54 \\
\hline P40415 & ВР3561 & $\begin{array}{l}\text { Putative peptidyl-prolyl cis-trans } \\
\text { isomerase }\end{array}$ & $P$ & Mice & + & unknown & 51,52 \\
\hline
\end{tabular}

AProtein ID was provided by UniProt (https://www.uniprot.org). ${ }^{B}$ Cene loci were provided by NCBI (https://www.ncbi.nlm.nih.gov). 'Subcellular localization was predicted by PSORT (https://psort.hgc.jp). ${ }^{D}$ The reported immunogenicity of the listed antigens was restricted to their ability in eliciting antibody response, with the exception of P0A339 (GroES). EP0A339 (GroES) was reported as a major B. pertussis protein dominating MHC-II-presented epitope repertoire in human antigen-presenting cells. C, cytoplasm; E, extracellular; OM, outer membrane; P, periplasm.

sities or dominant responses after vaccination were selected for protein identification by LC-MS (Figure 5, B and C; and Table 1). The range of antigens recognized by the antibodies in the respective vaccine groups is shown in Table 1. BPZE1 induced IgG to multiple antigens, including BrkA that is ubiquitously expressed by $B$. pertussis mediating complement evasion; GroEL that elicits partial protection against $B$. pertussis challenge (49); and PNPase and LivJ, previously shown to be immunogenic (Table 2). In contrast, aPV-elicited IgG was directed primarily against the vaccine components, especially FHA, PRN, and PT, in line with the ELISA results. The anti-FHA and anti-PRN IgG responses appeared as multiple IgG-binding protein spots (Figure 5B and Table 1), likely due to degradation, deamination, or posttranslational modifications (44).

The specificities of the IgA response induced by BPZE1 also identified a variety of antigens, such as RpsA (spot 03) and GroEL (spot 04) that were able to elicit both IgA and IgG responses, PRN (spot 13) and FHA (spot 18), as well as some potentially novel antigens such as WbpO (spot 17) and GroES (spot 27). In addition, we observed a "tailed" IgA-binding protein spot at lower molecular weight (spot 28) and although not analyzed in our study, it may represent lipooligosaccharide (44). aPVelicited IgA was directed against fewer antigens, such as PRN, BrkA, DnaK, and Enolase, the 3 latter of which are not included in the vaccine. We again speculate that this is the result of natural exposure to $B$. pertussis or other pathogens exhibiting the same antigens. Given the critical role of IgA in protection against pertussis, we analyzed some of the antigens that solely induced IgA responses (spots labeled with purple circles in Figure 5C). These antigens were DnaK, PPIase, SODb, DsbA, NusG, PpiB, PT S4, and GroES (Table 1). Their functions were noted using GO analysis (Supplemental Table 3). In addition to
PT, FHA, and PRN included in aPV, several other of the antigens we identified (Table 2) have previously been shown to be immunogenic in either animal or human studies (49-55).

BPZE1-induced opsonizing antibodies mediate neutrophil activation and killing. Since neutrophils are major innate effector cells clearing B. pertussis in vaccinated or convalescent mice and not in naive mice $(56,57)$, we assessed whether the antibodies induced by BPZE1 or aPV have different abilities to opsonize bacteria and mediate killing. To test this, we exposed purified human neutrophils to BPZE1 opsonized by $10 \%$ of pooled serum from either vaccine group. ROS and activation (CD11b, CD66, CD63, CD62L) and maturation markers (CD10) were measured. Neutrophils exposed to BPZE1 upregulated CD11b and CD10 and downregulated CD62L, whereas ROS production was not affected (Figure 6A). In contrast, neutrophils exposed to BPZE1 opsonized by baseline serum from the BPZE1 group showed a much higher level of ROS, as well as further increased expression of CD11b, CD66, CD63 and decreased expression of CD62L, likely driven by the opsonizing capacity of the preexisting antibodies. This effect was further amplified with serum collected 28 days after BPZE1 vaccination. ROS production especially was strongly upregulated (Figure 6A) and was serum dose-dependent (Figure 6B). Increased ROS production over baseline was also found with serum collected 6 and 12 months after vaccination, although at a lower level (Figure 6C).

Compared with aPV, BPZE1 has been shown to confer a significantly more efficient bacterial clearance upon challenge (21, 22). Since the bacterial clearance was largely mediated by phagocytic cells such as neutrophils, we speculated that BPZE1-induced antibodies have better opsonizing capacity to activate neutrophils than aPV-induced antibodies. We therefore performed the same assay by exposing neutrophils to BPZE1 in the presence of vaccine-induced antibodies. In order to compare the activi- 
A
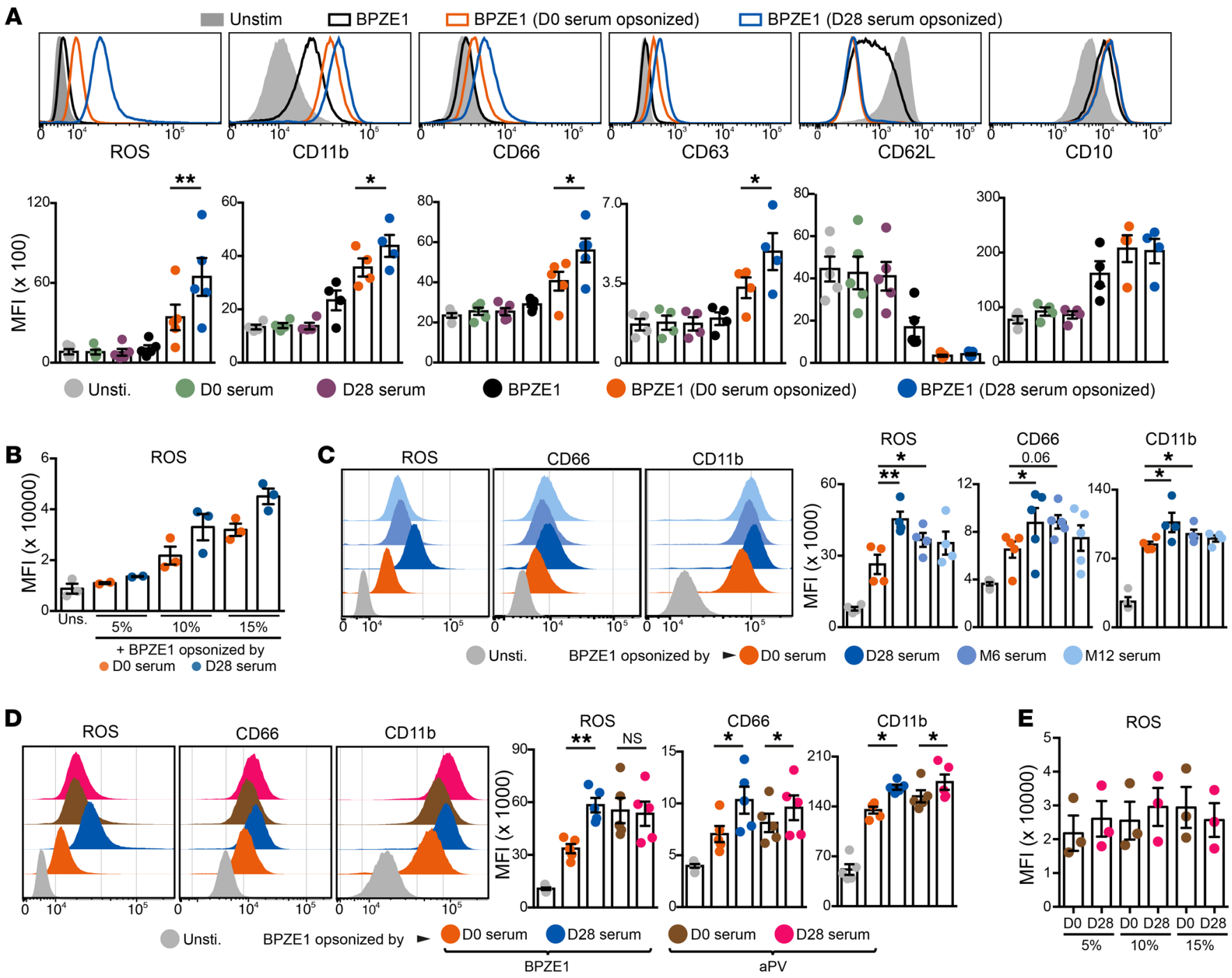

$\mathbf{F}$

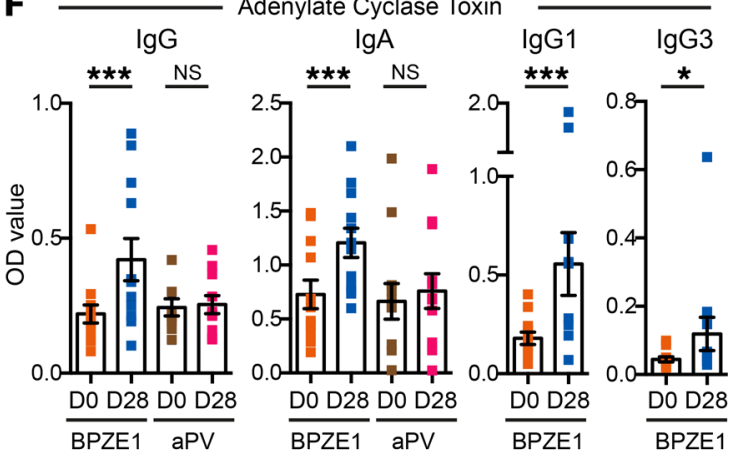

G

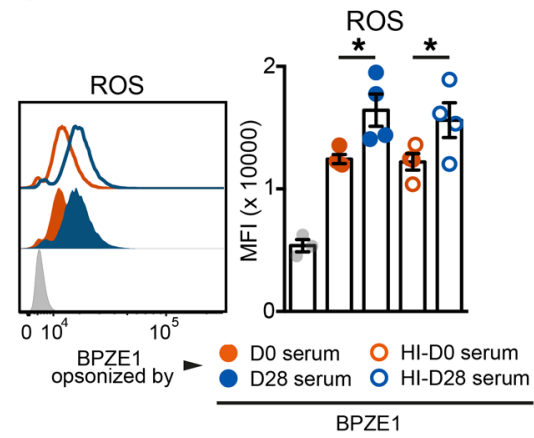

H

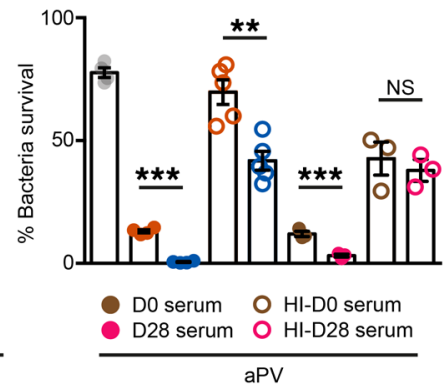

Figure 6. BPZE1 was superior to aPV at inducing opsonizing antibodies leading to enhanced neutrophil bactericidal activity. (A-E) Fresh human neutrophils $\left(10^{6}\right.$ cells) were infected with live BPZE1 (107 CFU) or BPZE1 opsonized by pooled serum of BPZE1 or aPV vaccinees for 1 hour. ROS production and expression of activation/maturation markers were evaluated. Compiled data are from at least 3 independent experiments. (A) $10 \%$ pooled serum of BPZE1 vaccinees $(n=12)$ were used. Cells without stimulation or cultured with pooled serum alone were used as control. MFI values of the indicated markers are shown. Two-tailed paired $t$ test was used. (B) Different concentrations of serum collected from BPZE1 vaccinees were evaluated. (C) Pooled serum of BPZE1 vaccinees collected at different time points were evaluated. Two-tailed paired $t$ test was used. (D) Pooled serum of BPZE1 or aPV vaccinees were evaluated and compared side by side. (E) Different concentrations of serum collected from aPV vaccinees were evaluated. (F) Antibody titers of IgG, IgA, IgG1, and IgG3 targeting ACT were measured in BPZE1 and aPV vaccinees $(n=12)$. Two-tailed Wilcoxon matched-pairs signed-rank test was used. (G) Neutrophils were infected with live BPZE1 opsonized by $10 \%$ pooled serum or $\mathrm{HI}$ serum of BPZE1 vaccinees for 1 hour. ROS production was evaluated. Compiled data from 4 independent experiments are shown. $(\mathbf{H})$ The effect of opsonization-mediated ROS production on the bactericidal activity of neutrophils was evaluated in vitro. Compiled data are from 4 independent experiments. Two-tailed paired $t$ test was used. ${ }^{*} P \leq 0.05,{ }^{* *} P \leq 0.01,{ }^{* * *} P \leq 0.001$. 
ties on an antibody basis, we normalized the serum between the groups to obtain equal quantity (i.e., $10 \%$ and $6 \%$ pooled serum of BPZE1 and aPV vaccines, respectively) based on the mean OD values of the IgG titers (Figure 4B). While both the BPZE1- and the aPV-induced antibodies activated neutrophils, indicated by elevated expression of CD11b and CD66, ROS production was only increased in the presence of BPZE1-induced antibodies (Figure 6D). Serum from aPV-immunized subjects failed to induce ROS production at all concentrations tested, up to 15\% (Figure 6E). Previous studies have shown that ACT released from B. pertussis inhibits ROS in neutrophils, whereas this could be reversed in the presence of ACT-specific antibodies (58-60). The BPZE1 vaccinees showed significantly increased titers of $\operatorname{IgG}$ and $\operatorname{IgA}$ against ACT after vaccination. IgG1 and IgG3 were again the main IgG subclasses induced by BPZE1 (Figure 6F). No IgG2 or IgG4 were elicited (data not shown). In contrast, aPV vaccination did not enhance the level of ACT-specific antibodies (Figure 6F). This may, at least partly, explain the inability of aPV-induced antibodies to stimulate ROS production.

Antibody-bacteria immune complexes can activate neutrophils through Fc-receptors. Additionally, complement molecules deposited on the bacterial surface as a result of antibody-dependent classical complement activation can activate neutrophils through complement receptors (61). To investigate whether ROS induction occurred through Fc-receptor- or complement-mediated neutrophil activation, we performed the same assay using heat-inactivated (HI) serum from BPZE1 vaccinees in which complement activity is destroyed. We found that HI serum-opsonized BPZE1 also induced ROS production in neutrophils (Figure 6G). This indicates that Fc-receptor-mediated opsonophagocytosis plays a dominant role in triggering ROS production by BPZE1-induced serum. Finally, we evaluated neutrophil killing activity after antibody-mediated bacterial opsonization. Enhanced killing by neutrophils was found using serum from either BPZE1 or aPV vaccinees to opsonize the bacteria (Figure $6 \mathrm{H}$ ). This may be due to a combination of both complement-mediated killing and neutrophil killing since the serum alone can kill B. pertussis through the activation of classical complement pathway, leading to the formation of membrane attack complex (Supplemental Figure 5). In the presence of HI serum from the BPZE1 vaccines, neutrophils showed a significantly better killing activity, whereas aPV-induced antibodies did not show this effect, consistent with their inability to stimulate ROS production (Figure 6H). Collectively, these data show that BPZE1 is superior to aPV in inducing effective opsonizing antibodies, leading to enhanced bactericidal activity of neutrophils. The induction of anti-ACT antibodies may be one mechanism explaining the better bacterial clearance conferred by BPZE1, as reported in animal studies $(21,22)$.

\section{Discussion}

Pertussis disease remains an important public health issue given the global rise of pertussis cases during the past decades (2). There is therefore a critical need for improved pertussis vaccines with high efficacy and low reactogenicity. The live BPZE1 vaccine has been tested in different animal models and demonstrated mucosal and systemic immune responses, long-lasting protection, and blocking of nasal colonization upon infection $(20,22)$. In human clinical trials, BPZE1 showed excellent safety profiles (24). In this study, we performed multiple assays to obtain mechanistic insight into the immune responses in humans generated by BPZE1 as compared with aPV. We found that a single intranasal administration of BPZE1 was able to induce well-detectable plasmablasts, activated cTfh 1 cells, vaccine-specific $\mathrm{CD} 4^{+}$cells, memory B cells and, ultimately, robust levels of Th1-type antibodies with potent bacterial opsonizing activity. We observed a much wider range of antigen specificities recognized by the antibodies elicited by BPZE1 compared with aPV. Importantly, potentially novel immunogenic antigens in BPZE1 were identified, which may be critical for generation of enhanced protection against B. pertussis disease and transmission.

With the full spectrum of antigens that BPZE1 expresses, it is not surprising that a wider repertoire of antibodies with multiple specificities can be induced as compared with the aPV. The identification of the immunogenic antigens of BPZE1 to which the antibody responses are directed is of particular importance because the correlates of protection against pertussis have yet to be definitively determined. Antibodies specific to the few aPV antigens are unlikely exclusive for protection since $B$. pertussis has a complex antigen composition and a large proportion of antigens overlap with other pathogens. In addition, B. pertussis isolates devoid of PRN, FHA, or PT have emerged as a result of bacterial evolution in response to aPV pressure $(9,10)$. We identified several immunogenic antigens in BPZE1 that have in fact been reported previously (Table 2). Notably, immunization of neonatal mice with purified GroEL was shown to confer protection against virulent $B$. pertussis challenge, albeit at a weaker level than that induced by PT (49). Immunization with recombinant iron superoxide dismutase together with monophosphoryl lipid A (MPLA) induced protective antibody and Th1 cell responses in mice (62). Vaccination with recombinant BrkA as a single component did not induce protection against pertussis. However, a 3-component aPV containing PT, FHA, and BrkA was able to generate more effective protection than an aPV containing only PT and FHA in mice (63).

Other antigens identified in our study consist of potentially novel antigens such as Q7VVC9 (spot 06), Q7VYH5 (spot 23), and Q7VSR6 (spot 31) located on the cell membrane. Moreover, antigens such as Q7VUV7 (spot 08), Q7VWW8 (spot 19), and Q7VXB3 (spot 20) are putative exported antigens. These antigens are absent in both the aPV and the wPV, since the production process of whole-cell vaccine typically discards the culture supernatant. These antigens are of particular interest since many antibacterial subunit vaccines like the Haemophilus influenzae type B vaccine and meningococcal group B vaccine contain surface exposed antigens or secreted antigens as components due to their direct interaction with host immune system (64-66). However, their role in induction of protective responses is not yet known.

Not only the breadth but also the function of the vaccine-induced antibodies determines the protective effect. Each IgG subclass possesses functional differences with respect to antigen binding, complement activation, opsonization, and triggering of effector phagocytes (67). Since innate effector cells such as neutrophils and macrophages play a key role in the clearance of $B$. pertussis (12), opsonizing antibodies that bind bacteria to form immune complexes and facilitate uptake and bacterial killing are 
central. Although uptake of bacteria was not evaluated directly in our study, we measured cell activation and ROS production (downstream effects of bacterial uptake). Antibodies from the individuals receiving BPZE1 showed stronger capacity to activate neutrophils and promote ROS production as compared with antibodies from aPV vaccinees, which further led to enhanced bactericidal activity. This was found to mainly depend on Fc-receptor ligation, which is in line with earlier studies (61). Antibodies from aPV vaccinees still activated neutrophils, but failed to enhance ROS production and promote neutrophil killing functions. It is plausible that this is influenced by the differences in the polarization of IgG subclasses induced by the different vaccines. BPZE1induced $\operatorname{Ig} G$ subclasses were only $\operatorname{IgG} 1$ and $\operatorname{IgG} 3$ that efficiently mediate opsonization, whereas aPV-induced IgG subclasses contained IgG2 and IgG4 that have no or limited opsonizing function. The mix of subclasses may compete for binding to the bacteria and interfere with uptake and signaling pathways regulating ROS production (67). Another potential explanation for the failure of aPV serum to stimulate ROS may be related to ACT released by $B$. pertussis, known to inhibit ROS production of neutrophils through cAMP signaling, which is critical for mediating bacterial evasion from immune responses (68). Only the BPZE1 vaccinees had induced robust anti-ACT antibodies, which could potentially have resulted in neutralization of ACT and alleviation of any inhibition of ROS production. Despite the fact that repeated aPV immunization elicits high antibody titers, these failed to promote ROS production and bacterial killing, which may help explain why $B$. pertussis can still colonize aPV-immunized individuals. The strong induction of ACT-specific antibodies by BPZE1 is therefore of particular importance. It has been shown that neither wPV nor aPV immunization can elicit anti-ACT antibodies in humans, whereas natural B. pertussis infection does (69). However, we observed that serum from aPV-immunized individuals before the boost immunization already showed strong ability to stimulate ROS production and promote neutrophil bactericidal activity. This is likely due to the high preexisting level of IgG directed to B. pertussis antigens from natural exposure since IgG-opsonized B. pertussis could enhance phagocytosis by neutrophils, thus promoting the subsequent bacterial killing $(61,70)$.

Differently skewed $\mathrm{T}$ cell responses also influence the quality of protection differently (36). Th1 and Th17 responses, especially when generated within the respiratory tract, contribute to effective protection against pertussis $(36,38)$. In mice, BPZE1 has been shown to generate a systemic Th1 response and a local Th17 response $(35,37)$. IL-17 and IFN- $\gamma$-producing resident memory T $\left(\mathrm{T}_{\mathrm{RM}}\right)$ cells also appeared at nasal sites upon BPZE1 immunization (37). We detected a systemic Th1 response induced by BPZE1. Although Th17 cells were not detectable systemically, they may perhaps still be elicited and only be present at airway mucosa. This was also indirectly evidenced by the strong induction of vaccine-specific IgA, which is closely associated with Th17 responses (71). Whether BPZE1 immunization can redirect the Th2-polarized, less protective immune responses induced by aPV will be essential to investigate in future studies. Initial exposure to aPV has been shown to lock the pertussis-specific immune responses to some degree (18), but there may be possibilities to shift the preformed Th2 signature to Th1/17 favoring pertussis immunity, especially at an early age when immune plasticity still remains. Also, it is not clear to what extent a systemic Th2 signature can suppress a Th1-prone vaccination event such as a colonization by BPZE1 at the local mucosa for 2 to 3 weeks.

In conclusion, this study provides potentially novel findings on increased antibody breadth and bacterial killing functions induced by BPZE1 compared with aPV. BPZE1 is now being tested in Phase II clinical trials in combination with aPV immunization (NCT03541499 and NCT03942406). Collectively, the data presented here will hopefully also enhance the overall basic understanding of the immunological mechanisms critical for pertussis protection and control.

\section{Methods}

Additional details are available in the Supplemental Materials and Methods.

Study subjects, immunization, and sample collection. Samples were obtained from 2 clinical trials registered at https://ClinicalTrials.gov under NCT02453048 (BPZE1 vaccine study) and NCT00870350 (Tdap vaccine study). Detailed information about the studies is available at https://ClinicalTrials.gov. In brief, the BPZE1 vaccine study was a randomized, double-blind, placebo-controlled, dose-escalating study. Healthy young adults (18-32 years of age) born in Sweden between 1984 and 1998, with anti-PT and/or PRN serum IgG titers of $20 \mathrm{IU} / \mathrm{mL}$ or more, were recruited. Notably, the majority of them were born during 1979-1996 when pertussis vaccination was suspended in Sweden. Therefore, any preexisting antibodies were most likely induced by natural infection with $B$. pertussis. We analyzed the responses in all study subjects $(n=12)$ enrolled in the group receiving a single intranasal immunization of the highest dose $\left(10^{9} \mathrm{CFU}\right)$ of BPZE1 in a volume of $0.4 \mathrm{~mL}$ per nostril. Samples from the placebo group ( $n=12$ subjects) were also analyzed in some assays as control. Peripheral venous blood was collected in BD Vacutainer CPT tubes at the day prior to vaccination and at days 4, 14, and 28, and months 6 and 12 after vaccination, followed by PBMCs isolation and serum collection. The Tdap vaccine study is a randomized, multicenter study in which the immunogenicity and safety of the tetanus, diphtheria, and acellular pertussis vaccine booster were evaluated $(72,73)$. In part of this clinical trial, adolescents (14-15 years of age) with a vaccination history of 3 primary immunizations of DTaP5 (Connaught HCP4DT) at 3,5, and 12 months of age plus a booster immunization of Tdap5 (Triaxis, Sanofi Pasteur) at 5.5 years of age received another booster dose of Tdap5 (Triaxis, Sanofi Pasteur). Serum samples were collected at 28 days after the final boost immunization and stored in the biobank at the Public Health Agency of Sweden. Samples ( $n=12$ subjects) were randomly selected by the biobank staff for analysis.

Antibody ELISA assay. BPZE1 and BP611-98 bacterial lysates were prepared as described in the Supplemental Materials and Methods. FHA, PT, PRN, FIM2/3, and ACT were purchased from List Biological Laboratories, USA or Kaketsuken, Japan. The 96-well half-area plates (Greiner Bio-One, Germany) were coated with bacterial lysates (500 ng/well) or a mixture of antigens (FHA, PT, PRN, FIM, $10 \mathrm{ng} /$ well each) overnight at $4^{\circ} \mathrm{C}$. In some assays, PT or ACT (10 ng/well) was used to coat the plates. Serum samples were diluted in PBS-T containing 0.2\% BSA (1:100 for IgG, 1:50 for IgA, IgG1 and IgG2, 1:10 for IgG3 and IgG4) prior to incubation at room temperature (RT) for 2 hours. After washing, total IgG was evaluated using HRP-conjugated 
mouse anti-human IgG for 1 hour (clone: G18-145, BD Biosciences). IgA and IgG subclasses were evaluated using mouse anti-human IgA (clone: G18-1, BD Biosciences), IgG1 (clone: G17-1, BD Biosciences), IgG2 (clone: G18-21, BD Biosciences), IgG3 (clone: HP-6050, SigmaAldrich), IgG4 (clone: G17-4, BD Biosciences) and further analyzed with HRP-conjugated sheep anti-mouse IgG (Jackson). TMB Substrate (Biolegend) was used for development, and the absorbance was read at $450 \mathrm{~nm}$ (minus $550 \mathrm{~nm}$ for wavelength correction). The limit of detection was an OD value of up to 2.5 .

2D PAGE. 2D PAGE was performed according to Apostolovic et al. (74). Briefly, bacterial lysates $(45 \mu \mathrm{g})$ were mixed with Destreak Rehydration Buffer and 0.5\% IPG Buffer pH 3-11 NL (GE Healthcare), up to a total volume of $125 \mu \mathrm{L}$. Immobiline DryStrips $\mathrm{pH}$ 3-11 NL, $7 \mathrm{~cm}$ (GE Healthcare) were rehydrated with the samples in a Reswell Tray (GE Healthcare) overnight at $4^{\circ} \mathrm{C}$ and run on Ettan IPGphor 3 Isoelectric Focusing system according to the protocol: 45 minutes at 300 $\mathrm{V}$ (Hold), 1 hour at $1000 \mathrm{~V}$ (Gradient), 1.5 hours at $5000 \mathrm{~V}$ (Gradient), 30 minutes at $5000 \mathrm{~V}$ (Hold). Then, proteins on the strip were reduced with $5 \mathrm{~mL}$ of equilibration buffer (6M urea, $75 \mathrm{mM}$ Tris- $\mathrm{HCl}$, pH 8.8, 29.3\% glycerol, 2\% SDS, 0.002\% bromophenol blue) containing 3\% DTT (Sigma-Aldrich) for 25 minutes, followed by alkylation with $5 \mathrm{~mL}$ of equilibration buffer containing 3\% iodoacetamide (Sigma-Aldrich) for 15 minutes in dark. Following this, the second dimension of separation was carried out on $12.5 \%$ hand-cast acrylamide gel in Hoefer Mighty Small II Mini Vertical Electrophoresis System using constant voltage of $180 \mathrm{~V}$. Protein spots were visualized by staining the gel with colloidal Coomassie Brilliant Blue solution according to the protocol (75).

Immunoblotting. After 2D PAGE, proteins were transferred to PVDF (Immobilon P $0.2 \mu \mathrm{m}$, MilliporeSigma) membrane in a TransBlot Turbo Transfer System (Bio-Rad) for 45 minutes under constant current (100 mA) using Towbin buffer (76). After complete transfer, the membrane was blocked in $5 \%$ skim milk overnight at $4^{\circ} \mathrm{C}$. For IgG detection, membrane was incubated with sera (1:100) pooled from all 12 vaccines in each group for 3 hours at room temperature. After washing with PBS-T for 15 minutes, the membrane was incubated with HRP-conjugated mouse anti-human IgG for 1 hour (clone: G18-145, $\mathrm{BD}$ Biosciences). For IgA detection, the membrane was incubated with pooled sera (1:20) overnight at $4^{\circ} \mathrm{C}$, followed by 3 washing steps and 1 hour of incubation with mouse anti-human IgA (clone: G18-1, BD Biosciences) and then with HRP-conjugated sheep anti-mouse IgG (515-035-003, Jackson). After washing step with PBS-T for 15 minutes, spots were visualized using Amersham ECL Prime Western Blotting Detection Reagent (GE Healthcare) and ChemiDoc MP Imaging System (Bio-Rad). Spot intensities, shown as average gray value, were quantified using Delta2D software (DECODON GmbH).

Flow cytometric analysis. Cells were collected, washed, and then stained with LIVE/DEAD Fixable Blue Dead Cells Stain Kit (Ther-
moFisher Scientific) prior to incubation with human FcR blocking reagent and antibody cocktails for 20 minutes at RT in the dark. Antibodies used in this study are listed in Supplemental Table 1. Flow cytometry analysis was carried out on LSRFortessa cell analyzer (BD Biosciences). Data were analyzed using FlowJo V.10.1 (Tree Star).

Statistics. Comparisons between different time points within the BPZE1, aPV, and placebo groups were analyzed using the 2-tailed paired nonparametric Wilcoxon's test. Comparisons between different groups were analyzed using the 2-tailed unpaired nonparametric Mann-Whitney test. The 2-tailed paired Student's $t$ test was used to analyze data from monocyte/neutrophil stimulation experiments and the neutrophil bactericidal assay. Correlation analysis between 2 parameters was performed using Pearson's correlation analysis. All statistical analyses were conducted by Prism Version 6.0 software (GraphPad), and the values are presented as mean \pm SEM in all figures. A $P$ value less than 0.05 was considered statistically significant.

Study approval. This study was approved by the Swedish Medical Product Agency (MPA) and the Stockholm Local Ethical Committee, and was performed according to the Declaration of Helsinki principles. All subjects gave written informed consent before inclusion in this study.

\section{Author contributions}

AL, DA, RT, MT, and KL designed research. AL, DA, MJ, FL, TT, and SO performed experiments. AL, FL, and CYW performed analysis. AL, RT, MvH, CL, MT, and KL discussed the data. KS, $\mathrm{KR}$, and MT provided BPZE1 vaccine. AL and KL wrote the manuscript. All authors provided critical review of the manuscript.

\section{Acknowledgments}

The authors thank Akos Vegvari at Proteomics Biomedicum for technical assistance with proteomics experiments. We also thank Kerstin Nyrén for providing BPZE1 and BP611-98 strains, and Andreas Mörner at the Public Health Agency of Sweden for providing serum samples from a Tdap clinical study. This project was supported by funds from ILiAD Biotechnologies and a grant from the Swedish Research Council (Vetenskapsrådet) (to KL). AL was supported by a grant from the China Scholarship Council and a PhD salary grant from Karolinska Institutet. DA was supported by the Konsul Th C Berg Foundation. MvH was supported by the Swedish Research Council (Vetenskapsrådet), the Stockholm County Council (ALF project), and the Heart-Lung Foundation.

Address correspondence to: Karin Loré, BioClinicum, J7:30, Akademiska stråket 1, 17177, Stockholm, Sweden. Phone: 46.0.8.524.876.72; Email:karin.lore@ki.se.

Frank Liang's present address is: Department of Microbiology and Immunology, University of Gothenburg, Gothenburg, Sweden.

\footnotetext{
1. Melvin JA, Scheller EV, Miller JF, Cotter PA. Bordetella pertussis pathogenesis: current and future challenges. Nat Rev Microbiol. 2014;12(4):274-288.

2. PERISCOPE Consortium. PERISCOPE: road towards effective control of pertussis. Lancet Infect Dis. 2019;19(5):e179-e186.

3. Cherry JD. Why do pertussis vaccines fail? Pediatrics. 2012;129(5):968-970.

4. Heininger $U$. The persistence of pertussis and
}

developments in vaccination strategies. Lancet Respir Med. 2015;3(9):668-670.

5. Witt MA, Arias L, Katz PH, Truong ET, Witt DJ. Reduced risk of pertussis among persons ever vaccinated with whole cell pertussis vaccine compared to recipients of acellular pertussis vaccines in a large US cohort. Clin Infect Dis. 2013;56(9):1248-1254.

6. Althouse BM, Scarpino SV. Asymptomatic trans- mission and the resurgence of Bordetella pertussis. BMC Med. 2015;13:146.

7. Kapil P, Merkel TJ. Pertussis vaccines and protective immunity. Curr Opin Immunol. 2019;59:72-78.

8. Xu Z, et al. Pertactin-Negative and Filamentous Hemagglutinin-Negative Bordetella pertussis, Australia, 2013-2017. Emerging Infect Dis. 2019;25(6):1196-1199.

9. Martin SW, et al. Pertactin-negative Bordetella 
pertussis strains: evidence for a possible selective advantage. Clin Infect Dis. 2015;60(2):223-227.

10. Williams MM, et al. Bordetella pertussis Strain Lacking Pertactin and Pertussis Toxin. Emerging Infect Dis. 2016;22(2):319-322.

11. Octavia $\mathrm{S}$, et al. Insight into evolution of Bordetella pertussis from comparative genomic analysis: evidence of vaccine-driven selection. Mol Biol Evol. 2011;28(1):707-715.

12. Higgs R, Higgins SC, Ross PJ, Mills KH. Immunity to the respiratory pathogen Bordetella pertussis. Mucosal Immunol. 2012;5(5):485-500.

13. Cherry JD, Gornbein J, Heininger U, Stehr K. A search for serologic correlates of immunity to Bordetella pertussis cough illnesses. Vaccine. 1998;16(20):1901-1906

14. Storsaeter J, Hallander HO, Gustafsson L, Olin P. Levels of anti-pertussis antibodies related to protection after household exposure to Bordetella pertussis. Vaccine. 1998;16(20):1907-1916.

15. Plotkin SA. Correlates of protection induced by vaccination. Clin Vaccine Immunol. 2010;17(7):1055-1065.

16. Chen X, Bailleux F, Desai K, Qin L, Dunning AJ. A threshold method for immunological correlates of protection. BMC Med Res Methodol. 2013;13:29.

17. Cassone A, Mastrantonio P, Ausiello CM. Are only antibody levels involved in the protection against pertussis in acellular pertussis vaccine recipients? J Infect Dis. 2000;182(5):1575-1577.

18. da Silva Antunes R, et al. Th1/Th17 polarization persists following whole-cell pertussis vaccination despite repeated acellular boosters. J Clin Invest. 2018;128(9):3853-3865.

19. Huang CC, et al. Experimental Whooping cough. N Engl JMed.1962;266:105-111.

20. Mielcarek N, et al. Live attenuated B. pertussis as a single-dose nasal vaccine against whooping cough. PLoS Pathog. 2006;2(7):e65.

21. Feunou PF, Kammoun H, Debrie AS, Mielcarek $\mathrm{N}$, Locht $\mathrm{C}$. Long-term immunity against pertussis induced by a single nasal administration of live attenuated B. pertussis BPZE1. Vaccine. 2010;28(43):7047-7053.

22. Locht $C$, et al. Live Attenuated Pertussis Vaccine BPZE1 Protects Baboons Against Bordetella pertussis Disease and Infection. J Infect Dis. 2017;216(1):117-124.

23. Warfel JM, Zimmerman LI, Merkel TJ. Acellular pertussis vaccines protect against disease but fail to prevent infection and transmission in a nonhuman primate model. Proc Natl Acad Sci USA. 2014;111(2):787-792.

24. Thorstensson R, et al. A phase I clinical study of a live attenuated Bordetella pertussis vaccine-BPZE1; a single centre, double-blind, placebocontrolled, dose-escalating study of BPZE1 given intranasally to healthy adult male volunteers. PLoS One. 2014;9(1):e83449.

25. Carbonetti NH. Pertussis leukocytosis: mechanisms, clinical relevance and treatment. Pathog Dis. 2016;74(7):ftw087.

26. Fink K. Origin and Function of Circulating Plasmablasts during Acute Viral Infections. Front Immunol. 2012;3:78.

27. Nakaya HI, et al. Systems biology of vaccination for seasonal influenza in humans. Nat Immunol. 2011;12(8):786-795.
28. Wrammert J, et al. Rapid and massive virus-specific plasmablast responses during acute dengue virus infection in humans. J Virol. 2012;86(6):2911-2918.

29. Cárdeno A, Magnusson MK, Quiding-Järbrink $\mathrm{M}$, Lundgren A. Activated T follicular helper-like cells are released into blood after oral vaccination and correlate with vaccine specific mucosal B-cell memory. Sci Rep. 2018;8(1):2729.

30. Schmitt N, Bentebibel SE, Ueno H. Phenotype and functions of memory Tfh cells in human blood. Trends Immunol. 2014;35(9):436-442.

31. Herati RS, et al. Successive annual influenza vaccination induces a recurrent oligoclonotypic memory response in circulating $\mathrm{T}$ follicular helper cells. Sci Immunol. 2017;2(8):eaag2152.

32. Lindgren $G$, et al. Induction of Robust B Cell Responses after Influenza mRNA Vaccination Is Accompanied by Circulating Hemagglutinin-Specific ICOS+ PD-1+ CXCR3+ T Follicular Helper Cells. Front Immunol. 2017;8:1539.

33. Bentebibel SE, et al. Induction of ICOS+CXCR3+CXCR5+ TH cells correlates with antibody responses to influenza vaccination. Sci Transl Med. 2013;5(176):176ra32.

34. Zhang J, et al. Circulating CXCR3 ${ }^{+}$Tfh cells positively correlate with neutralizing antibody responses in HCV-infected patients. Sci Rep. 2019;9(1):10090.

35. Skerry CM, Mahon BP. A live, attenuated Bordetella pertussis vaccine provides long-term protection against virulent challenge in a murine model. Clin Vaccine Immunol. 2011;18(2):187-193.

36. Fedele G, Cassone A, Ausiello CM. T-cell immune responses to Bordetella pertussis infection and vaccination. Pathog Dis. 2015;73(7):ftv051.

37. Solans L, et al. IL-17-dependent SIgA-mediated protection against nasal Bordetella pertussis infection by live attenuated BPZE1 vaccine. Mucosal Immunol. 2018;11(6):1753-1762.

38. Wilk MM, Borkner L, Misiak A, Curham L, Allen AC, Mills KHG. Immunization with whole cell but not acellular pertussis vaccines primes $\mathrm{CD} 4$ $\mathrm{T}_{\mathrm{RM}}$ cells that sustain protective immunity against nasal colonization with Bordetella pertussis. Emerg Microbes Infect. 2019;8(1):169-185.

39. Leef M, Elkins KL, Barbic J, Shahin RD. Protective immunity to Bordetella pertussis requires both B cells and CD4(+) T cells for key functions other than specific antibody production. J Exp Med. 2000;191(11):1841-1852.

40. Feunou PF, Bertout J, Locht C. T- and B-cell-mediated protection induced by novel, live attenuated pertussis vaccine in mice. Cross protection against parapertussis. PLoS One. 2010;5(4):e10178.

41. Dirix V, et al. Both $\mathrm{CD}^{+}{ }^{+}$and $\mathrm{CD} 8^{+}$lymphocytes participate in the IFN- $\gamma$ response to filamentous hemagglutinin from Bordetella pertussis in infants, children, and adults. Clin Dev Immunol. 2012;2012:795958.

42. Rieber N, Graf A, Hartl D, Urschel S, Belohradsky $\mathrm{BH}$, Liese J. Acellular pertussis booster in adolescents induces Th1 and memory CD $8+\mathrm{T}$ cell immune response. PLoS One. 2011;6(3):e17271.

43. Carbonetti NH, Khelef N, Guiso N, Gross R. A phase variant of Bordetella pertussis with a mutation in a new locus involved in the regulation of pertussis toxin and adenylate cyclase toxin expression. J Bacteriol. 1993;175(20):6679-6688.

44. Raeven RH, et al. Immunoproteomic Profiling of Bordetella pertussis Outer Membrane Vesicle Vaccine Reveals Broad and Balanced Humoral Immunogenicity. J Proteome Res. 2015;14(7):2929-2942.

45. Le T, et al. Immune responses and antibody decay after immunization of adolescents and adults with an acellular pertussis vaccine: the APERT Study. Jinfect Dis. 2004;190(3):535-544.

46. Hendrikx LH, et al. Different IgG-subclass distributions after whole-cell and acellular pertussis infant primary vaccinations in healthy and pertussis infected children. Vaccine. 2011;29(40):6874-6880.

47. Rowe J, et al. Antigen-specific responses to diphtheria-tetanus-acellular pertussis vaccine in human infants are initially Th2 polarized. Infect Immun. 2000;68(7):3873-3877.

48. Debrie AS, et al. Construction and evaluation of Bordetella pertussis live attenuated vaccine strain BPZE1 producing Fim3. Vaccine. 2018;36(11):1345-1352.

49. Burns DL, Gould-Kostka JL, Kessel M, Arciniega JL. Purification and immunological characterization of a GroEL-like protein from Bordetella pertussis. Infect Immun. 1991;59(4):1417-1422.

50 . West $\mathrm{R}$, et al. A rapid method for capture and identification of immunogenic proteins in Bordetella pertussis enriched membranes fractions: a fast-track strategy applicable to other microorganisms. J Proteomics. 2012;75(6):1966-1972.

51. Altindiş E, et al. Immunoproteomic analysis of Bordetella pertussis and identification of new immunogenic proteins. Vaccine. 2009;27(4):542-548.

52. Tefon BE, Maass S, Ozcengiz E, Becher D, Hecker $\mathrm{M}$, Ozcengiz G. A comprehensive analysis of Bordetella pertussis surface proteome and identification of new immunogenic proteins. Vaccine. 2011;29(19):3583-3595.

53. Raeven RHM, et al. Antibody Specificity Following a Recent Bordetella pertussis Infection in Adolescence Is Correlated With the Pertussis Vaccine Received in Childhood. Front Immunol. 2019;10:1364.

54. Del Giudice G, et al. Priming to heat shock proteins in infants vaccinated against pertussis. JImmunol.1993;150(5):2025-2032.

55. Stenger RM, et al. Bordetella pertussis proteins dominating the major histocompatibility complex class II-presented epitope repertoire in human monocyte-derived dendritic cells. Clin Vaccine Immunol. 2014;21(5):641-650.

56. Andreasen C, Carbonetti NH. Role of neutrophils in response to Bordetella pertussis infection in mice. Infect Immun. 2009;77(3):1182-1188.

57. Eby JC, Hoffman CL, Gonyar LA, Hewlett EL. Review of the neutrophil response to Bordetella pertussis infection. Pathog Dis. 2015;73(9):ftv081.

58. Eby JC, Gray MC, Hewlett EL. Cyclic AMP-mediated suppression of neutrophil extracellular trap formation and apoptosis by the Bordetella pertussis adenylate cyclase toxin. Infect Immun. 2014;82(12):5256-5269.

59. Confer DL, Eaton JW. Phagocyte impotence caused by an invasive bacterial adenylate cyclase. 


\section{CLINICAL MEDICINE}

Science. 1982;217(4563):948-950.

60. Mobberley-Schuman PS, Connelly B, Weiss AA. Phagocytosis of Bordetella pertussis incubated with convalescent serum. JInfect Dis. 2003;187(10):1646-1653.

61. Hellwig SM, van Oirschot HF, Hazenbos WL, van Spriel AB, Mooi FR, van De Winkel JG. Targeting to Fcgamma receptors, but not CR3 (CD11b/ CD18), increases clearance of Bordetella pertussis. J Infect Dis. 2001;183(6):871-879.

62. Yılmaz Ç, Apak A, Özcengiz E, Özcengiz G. Immunogenicity and protective efficacy of recombinant iron superoxide dismutase protein from Bordetella pertussis in mice models. Microbiol Immunol. 2016;60(11):717-724.

63. Marr N, Oliver DC, Laurent V, Poolman J, Denoël P, Fernandez RC. Protective activity of the Bordetella pertussis BrkA autotransporter in the murine lung colonization model. Vaccine. 2008;26(34):4306-4311.

64. Grandi G. Bacterial surface proteins and vaccines. F1000 Biol Rep. 2010;2:36.

65. Garmory HS, Titball RW. ATP-binding cassette transporters are targets for the development of antibacterial vaccines and therapies. Infect Immun. 2004;72(12):6757-6763.
66. Hilleman MR. Vaccines in historic evolution and perspective: a narrative of vaccine discoveries. Vaccine. 2000;18(15):1436-1447.

67. Vidarsson G, Dekkers G, Rispens T. IgG subclasses and allotypes: from structure to effector functions. Front Immunol. 2014;5:520.

68. Cerny O, Anderson KE, Stephens LR, Hawkins PT, Sebo P. cAMP Signaling of Adenylate Cyclase Toxin Blocks the Oxidative Burst of Neutrophils through Epac-Mediated Inhibition of Phospholipase C Activity. JImmunol. 2017;198(3):1285-1296.

69. Cherry JD, Xing DX, Newland P, Patel K, Heininger U, Corbel MJ. Determination of serum antibody to Bordetella pertussis adenylate cyclase toxin in vaccinated and unvaccinated children and in children and adults with pertussis. Clin Infect Dis. 2004;38(4):502-507.

70. Hellwig SM, Rodriguez ME, Berbers GA, van de Winkel JG, Mooi FR. Crucial role of antibodies to pertactin in Bordetella pertussis immunity. J Infect Dis. 2003;188(5):738-742.

71. Christensen D, Mortensen R, Rosenkrands I, Dietrich J, Andersen P. Vaccine-induced Th17 cells are established as resident memory cells in the lung and promote local IgA responses. Mucosal Immunol. 2017;10(1):260-270.
72. Jahnmatz M, Ljungman M, Netterlid E, Jenmalm MC, Nilsson L, Thorstensson R. Pertussis-specific memory B-cell and humoral IgG responses in adolescents after a fifth consecutive dose of acellular pertussis vaccine. Clin Vaccine Immunol. 2014;21(9):1301-1308.

73. Carlsson RM, et al. Two consecutive randomized controlled pertussis booster trials in children initially vaccinated in infancy with an acellular vaccine: The first with a five-component Tdap vaccine to 5-year olds and the second with fiveor monocomponent Tdap vaccines at age 14-15 years. Vaccine. 2015;33(31):3717-3725.

74. Apostolovic D, Tran TA, Hamsten C, Starkhammar M, Cirkovic Velickovic T, van Hage M. Immunoproteomics of processed beef proteins reveal novel galactose- $\alpha$-1,3-galactose-containing allergens. Allergy. 2014;69(10):1308-1315.

75. Dyballa N, Metzger S. Fast and sensitive colloidal coomassie G-250 staining for proteins in polyacrylamide gels. J Vis Exp. 2009;(30):1431.

76. Towbin H, Staehelin T, Gordon J. Electrophoretic transfer of proteins from polyacrylamide gels to nitrocellulose sheets: procedure and some applications. Proc Natl Acad Sci USA. 1979;76(9):4350-4354. 